ARTICLE

\title{
OTULIN maintains skin homeostasis by controlling keratinocyte death and stem cell identity
}

\author{
Esther Hoste (1) 1,2,3,7,8凶 , Kim Lecomte ${ }^{1,2,7}$, Karl Annusver (1) 4, Niels Vandamme ${ }^{1,5}$, Jana Roels (1) 1,5,
} Sophia Maschalidi (1) 1,2, Lien Verboom (10 1,2, Hanna-Kaisa Vikkula1,2, Mozes Sze ${ }^{1,2}$, Lisette Van Hove ${ }^{1,2}$, Kevin Verstaen ${ }^{1,5}$, Arne Martens ${ }^{1,2}$, Tino Hochepied ${ }^{1,2}$, Yvan Saeys ${ }^{1,5}$, Kodi Ravichandran 1,2,6, Maria Kasper ${ }^{4}$ \& Geert van Loo (1,2,3,8凶

OTULIN is a deubiquitinase that specifically cleaves linear ubiquitin chains. Here we demonstrate that the ablation of Otulin selectively in keratinocytes causes inflammatory skin lesions that develop into verrucous carcinomas. Genetic deletion of Tnfr1, knockin expression of kinase-inactive Ripk1 or keratinocyte-specific deletion of Fadd and Mlkl completely rescues mice with OTULIN deficiency from dermatitis and tumorigenesis, thereby identifying keratinocyte cell death as the driving force for inflammation. Single-cell RNA-sequencing comparing non-lesional and lesional skin reveals changes in epidermal stem cell identity in OTULIN-deficient keratinocytes prior to substantial immune cell infiltration. Keratinocytes lacking OTULIN display a type-1 interferon and IL-1 $\beta$ response signature, and genetic or pharmacologic inhibition of these cytokines partially inhibits skin inflammation. Finally, expression of a hypomorphic mutant Otulin allele, previously shown to cause OTULIN-related autoinflammatory syndrome in humans, induces a similar inflammatory phenotype, thus supporting the importance of OTULIN for restraining skin inflammation and maintaining immune homeostasis.

\footnotetext{
${ }^{1}$ VIB Center for Inflammation Research, Ghent, Belgium. ${ }^{2}$ Department of Biomedical Molecular Biology, Ghent University, Ghent, Belgium. ${ }^{3}$ Cancer Research Institute Ghent (CRIG), Ghent, Belgium. ${ }^{4}$ Department of Cell and Molecular Biology, Karolinska Institutet, Stockholm, Sweden. ${ }^{5}$ Department of Applied Mathematics, Computer Sciences and Statistics, Ghent University, Ghent, Belgium. ${ }^{6}$ Center for Cell Clearance and Department of Microbiology, Immunology and Cancer Biology, University of Virginia, Charlottesville, Virginia, USA. ${ }^{7}$ These authors contributed equally: Esther Hoste, Kim Lecomte. ${ }^{8}$ These authors

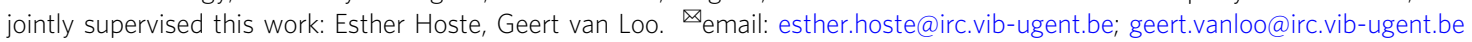


T he skin protects our body from external insults and against dehydration. Keratinocytes, the epithelial cells of the skin, undergo a tightly regulated differentiation program that enables the formation of a fully functional epidermal permeability barrier. Keratinocyte stem cells replace cells that have been lost through normal differentiation or programmed cell death. In recent years, a wide variety of keratinocyte stem cells have been identified that reside in different skin compartments. However, in pathophysiological conditions, such as in inflammation, wounding, or tumorigenesis, stem cells can display a tremendous plasticity and perform functions that are not part of their homeostatic repertoire ${ }^{1-3}$. The molecular mechanisms underlying stem cell plasticity in different pathophysiological skin states are largely unknown.

Linear ubiquitination represents a post-translational modification that is characterized by the addition of methionine (M1)linked ubiquitin chains on protein substrates by the linear ubiquitin chain assembly complex (LUBAC) ${ }^{4}$. LUBAC, which consists of the proteins SHARPIN, HOIP, and HOIL-1, is the only currently known E3 ligase complex responsible for M1 ubiquitin chain formation ${ }^{5-7}$, and mice deficient for SHARPIN or lacking HOIL-1 or HOIP in keratinocytes develop severe dermatitis ${ }^{8-10}$. Indeed, LUBAC-mediated linear ubiquitination controls the activation of the pro-inflammatory NF- $\mathrm{KB}$ pathway, but also prevents tumor necrosis factor receptor 1 (TNFR1)-mediated cell death ${ }^{4,11}$. OTULIN (OTU deubiquitinase with linear linkage specificity, also known as Fam105b or Gumby) is the sole deubiquitinase that exhibits a unique affinity for linear ubiquitin chains ${ }^{12-14}$. Patients harboring loss-of-function mutations in OTULIN develop a severe auto-inflammatory disease with skin involvement, termed ORAS (OTULIN-related auto-inflammatory syndrome, also known as otulipenia) ${ }^{15,16}$. Mice deficient for OTULIN or expressing a catalytically inactive OTULIN mutant die midgestation as a result of aberrant cell death mediated by TNFR1-signaling and receptor-interacting protein kinase 1 (RIPK1) kinase activity ${ }^{13,17}$. OTULIN directly binds to HOIP, and downregulation of OTULIN results in enhanced M1-linked ubiquitination of LUBAC and its substrates. These findings indicate that OTULIN promotes LUBAC activity by inhibiting LUBAC autoubiquitination and degradation ${ }^{17-20}$.

Recent data point to the fact that cell death and inflammation are intricately linked, and cell death mechanisms have been shown to initiate inflammatory responses ${ }^{21}$. The balance between pro-inflammatory gene activation and cell death relies on signal transduction by death receptors, such as TNFR $1^{21,22}$. The binding of TNF to TNFR1 induces the formation of the TNFR1 signaling complex, also termed complex I. Various adaptor proteins are sequentially recruited into this complex, resulting in the activation of pro-inflammatory NF- $\mathrm{KB}$ and MAPK signaling. Ubiquitination of distinct proteins in this complex is paramount for its assembly and downstream signaling. However, TNF can also induce inflammation by promoting cell death. In these circumstances, a different molecular complex is assembled, resulting in the formation of an apoptosis-inducing complex IIa, consisting of FADD (Fas-associated death domain) and caspase-8, or complex IIb, which relies on FADD and RIPK1 enzymatic activity, or in the formation of a necroptosis-inducing complex (termed necrosome) that depends on RIPK1 and RIPK3 kinase-activity and subsequent phosphorylation of MLKL (mixed lineage kinase domain-like $)^{21,22}$. Genetic studies in mice have revealed that defects in proper cell death regulation may induce severe inflammatory skin phenotypes caused by keratinocyte apoptosis and necroptosis, demonstrating that keratinocyte death is a potent trigger of skin inflammation and pathology $y^{23}$.

Here, we set out to investigate the importance of OTULINmediated linear deubiquitination for skin homeostasis by selectively deleting OTULIN in keratinocytes ( $\left.\Delta^{\mathrm{Ker}} \mathrm{OTULIN}\right)$ in

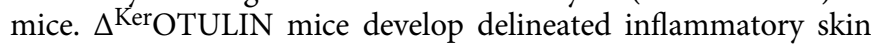
lesions from young age on that progress into verrucous carcinomas. Through genetic and pharmacological intervention studies, and by performing single-cell analysis on lesional and non-

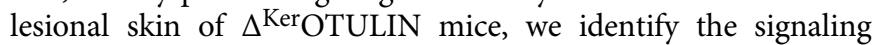
pathways through which these lesions appear, allowing us to get new insights on the molecular events that regulate skin homeostasis and mediate skin inflammation.

\section{Results}

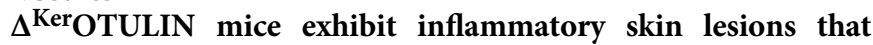
develop into verrucous carcinomas. To investigate the role of OTULIN in the epidermis, Otulin ${ }^{\text {fl/fl }}$ mice $^{24}$ were crossed to the Keratin-14 Cre line to enable Cre-mediated recombination and OTULIN deletion selectively in keratinocytes ${ }^{25}$. Immunoblot analysis of primary keratinocyte cultures isolated from

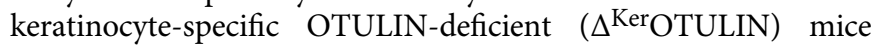
revealed efficient deletion of OTULIN (Supplementary Fig. 1a). $\Delta^{\mathrm{Ker} O T U L I N}$ mice were born with normal Mendelian segregation, but developed delineated inflamed skin lesions on back and

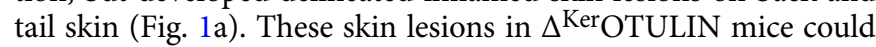
already be observed from postnatal day (P) 6 onwards (Supplementary Fig. 1b). Skin pathology was confirmed by histology on lesional back skin from adult 7 -week old $\Delta^{\text {KerOTULIN mice }}$ revealing marked epidermal hyperplasia and melanophagy (Fig. 1b). These inflammatory skin lesions progressively developed into verrucous carcinoma (Fig. 1b), defined as a welldifferentiated variant of squamous cell carcinoma with minimal metastatic potential ${ }^{26}$, and mice had to be sacrificed prior to tumor formation due to ethical concerns. Skin inflammation in

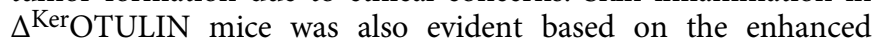
epidermal thickness of lesional skin of these mice, while the epidermis of non-lesional skin was not thickened and was comparable to the skin of control (OTULIN ${ }^{\mathrm{fl} / \mathrm{fl}}$ ) littermate mice

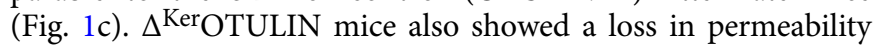
barrier integrity in lesional skin, as assessed by transepidermal water loss (TEWL) measurements (Fig. 1d). Dermatitis in $\Delta^{\text {Ker- }}$ OTULIN skin was further confirmed by the marked presence of

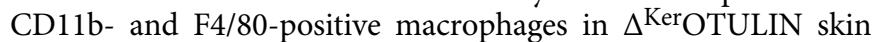
lesions (Fig. 1e), and aberrant keratinocyte differentiation could be demonstrated in both lesional and non-lesional skin of $\Delta^{\text {Ker- }}$ OTULIN mice based on abnormal Keratin-6 (K6) and filaggrin staining of skin sections (Fig. 1e). $\Delta^{\mathrm{Ker} O T U L I N}$ skin also exhibited substantial hypersebacea relative to the skin of control mice, as assessed by Oil Red O staining (Supplementary Fig. 1c).

Quantitative PCR analysis on epidermal tail lysates showed an increase in the expression levels of interleukin (IL)-4 and -13, which are both linked to epidermal barrier function ${ }^{27}$, the proinflammatory cytokines TNF and IL-6, the chemokine MCP-1 (Monocyte Chemoattractant Protein-1, also known as CCL2), and the antimicrobial peptide S100A8 (Fig. 1f) in $\Delta^{\mathrm{Ker} O T U L I N}$ skin compared to control skin, confirming the loss of barrier integrity and the inflammatory condition of $\Delta^{\text {Ker }}$ TTULIN skin. $\Delta^{\mathrm{Ker} O T U L I N}$ mice also exhibited enhanced circulating levels of IL-6, TNF, MCP-1, and IL-17 relative to control mice (Fig. 1g), indicating systemic inflammation, which was also apparent by the bigger size of the skin-draining lymph nodes in $\Delta^{\mathrm{Ker} O T U L I N}$ mice compared to control mice (Supplementary Fig. 1d). In agreement with the inflammatory condition of $\Delta^{\mathrm{Ker} O T U L I N}$ skin, immunoblotting on epidermal tail lysates revealed an enhanced NF- $\mathrm{KB}$ response, as evidenced by the reduced level of

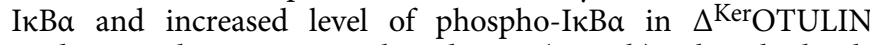
epidermis relative to control epidermis (Fig. 1h). Also, the levels of M1-linked ubiquitin chains were markedly increased in 
a.
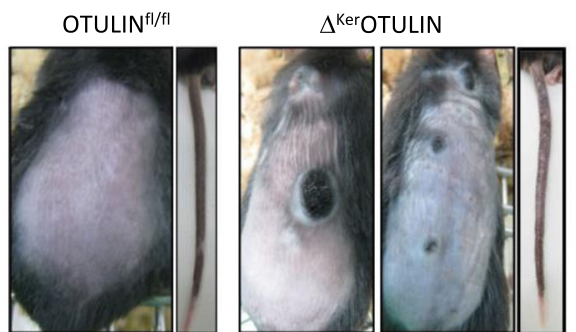

c.

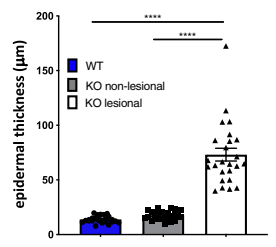

d.

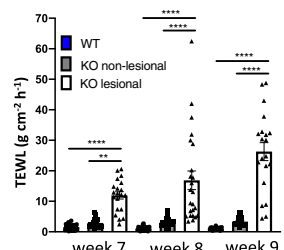

e.

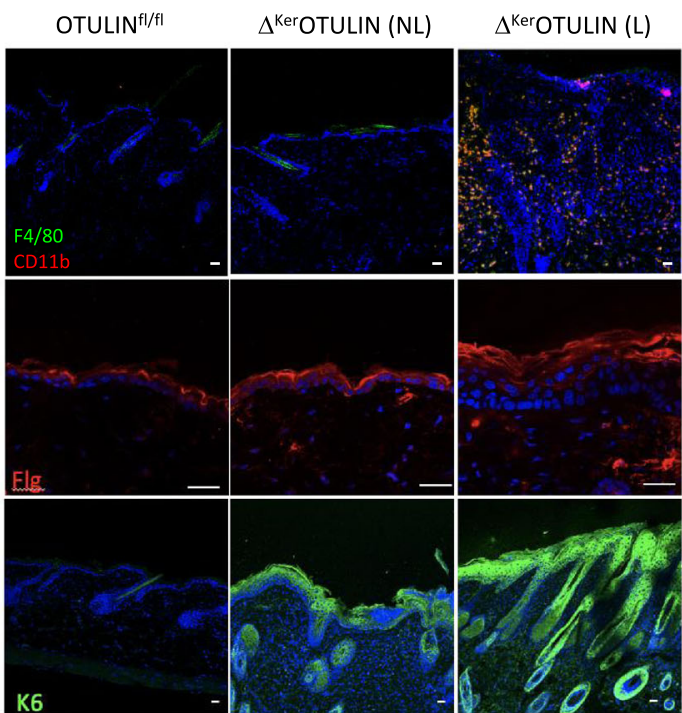

g.

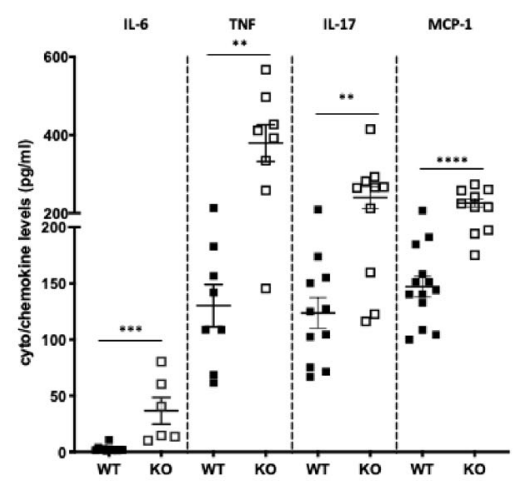

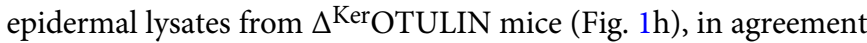
with the function of OTULIN as an M1 ubiquitin-specific deubiquitinase. Together, these data demonstrate the development of a strong but delineated dermatitis in mice that lack OTULIN in keratinocytes, suggesting that proper regulation of LUBAC-mediated linear ubiquitination is needed in order to maintain skin homeostasis.
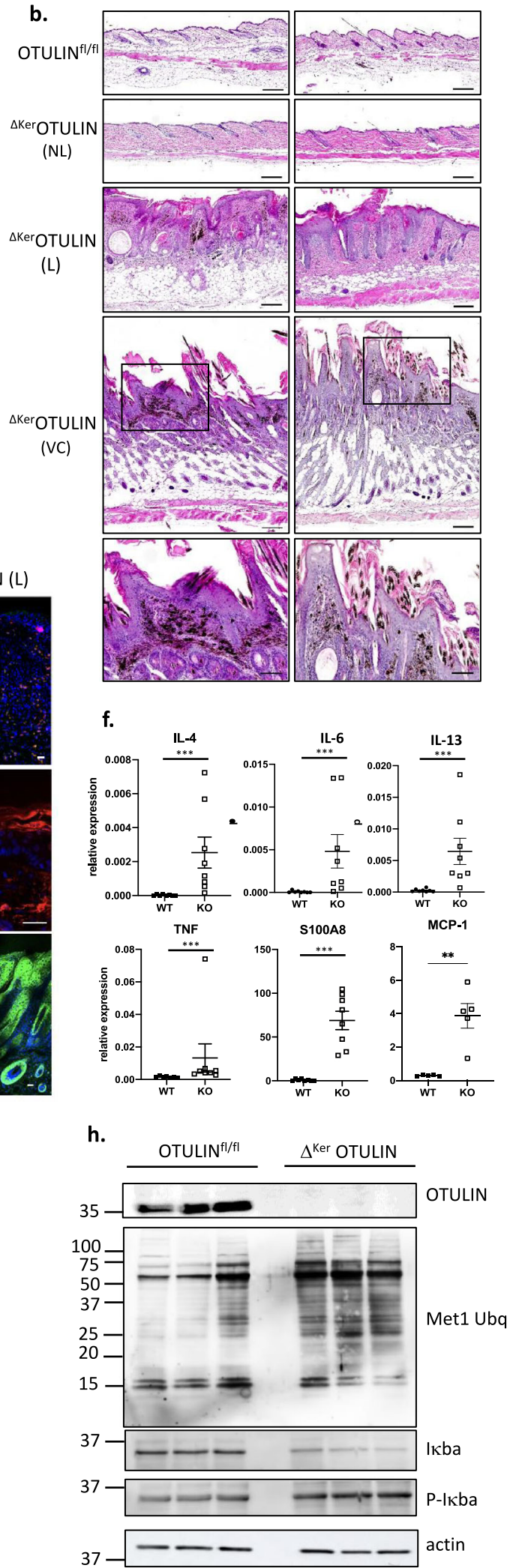

Ablation of OTULIN in keratinocytes results in enhanced epidermal stem cell proliferation and cell death. LUBACmediated linear ubiquitination proved to be important for the prevention of inflammation-induced cell death in the $\operatorname{skin}^{8}$. OTULIN has also been recognized for its role in limiting inflammatory cell death $17,24,28,29$. Therefore, we quantified the amount of cleaved caspase-3-positive apoptotic cells in 
Fig. $1 \Delta$ KerOTULIN mice exhibit delineated inflammatory skin lesions that develop into verrucous carcinomas. a Representative images of the back skin

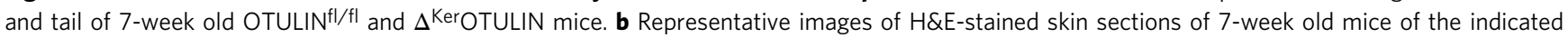

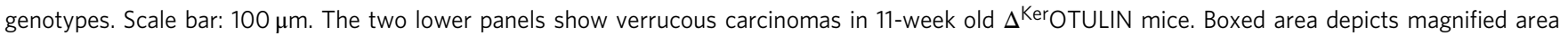
shown below. Scale bar for magnified areas: $200 \mu \mathrm{m}$. NL non-lesional; L lesional; VC verrucous carcinoma. c Epidermal thickness quantification at 7 weeks of age ( $n=5$ per condition; ${ }^{\star \star \star \star} p<0.0001$, One-way ANOVA with multiple comparisons). Data represent means \pm SEM. d Trans-epidermal water loss (TEWL) measurements at week 7, 8 and 9 of age ( $n=5$ mice per condition; ${ }^{\star *} p=0.0019 ;{ }^{\star \star \star *} p<0.0001$, One-way ANOVA with multiple comparisons). Data represent means \pm SEM. e Immunofluorescent staining of skin sections from 7-week old OTULIN ${ }^{f l / f l}$ mice and non-lesional (NL) and lesional (L) skin

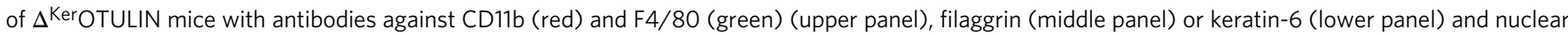
staining with Dapi. Each staining was performed on $n=5$ mice per condition. Scale bar: $25 \mu \mathrm{m}$. f Relative mRNA expression of IL-4, IL-6, IL-13, TNF,

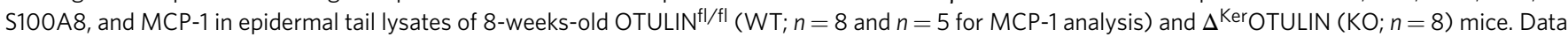
represent means \pm SEM. ${ }^{\star \star \star} p<0.001$; Mann-Whitney two-sided testing). $\mathbf{g}$ Levels of IL-6, TNF, IL-17, and MCP-1 in serum of 8-weeks-old OTULIN ${ }^{\mathrm{fl} / f \mathrm{fl}}$

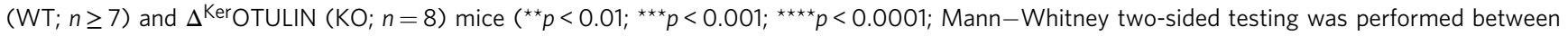

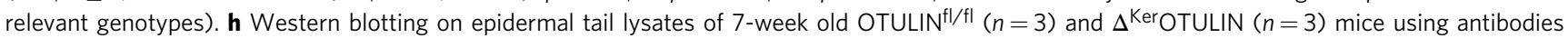

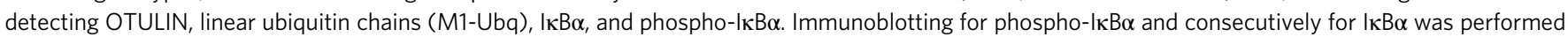
on the same blot. Molecular weight marker units are in kilodalton (kD). Anti-actin is shown as loading control. The representative image is shown for three independent experiments.

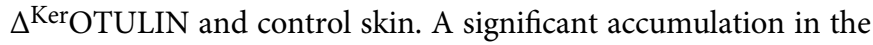
number of apoptotic cells could be demonstrated in non-lesional $\Delta^{\mathrm{Ker} O T U L I N}$ skin compared to control skin, which was even

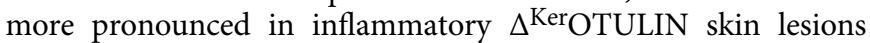
(Fig. 2a, b; Supplementary Fig. 2a). Caspase-3 cleavage was confirmed by immunoblotting on epidermal tail lysates from

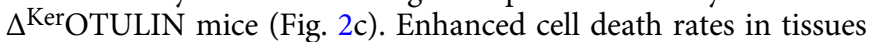
are often accompanied by compensatory cell proliferation. Analysis of $\Delta{ }^{\mathrm{Ker} O T U L I N}$ skin sections also showed a marked increase in keratinocyte proliferation in both lesional and non-lesional skin regions, as evidenced by Ki67 staining (Fig. 2a, b; Supplementary Fig. 2b). In agreement, we assessed keratinocyte pro-

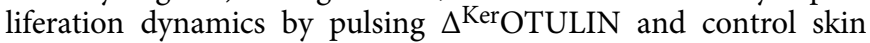
with the nucleotide analog EdU (5-ethynyl-2'-deoxyuridine) for $3 \mathrm{~h}$ prior to analysis. Wholemount immunofluorescence of hair follicles revealed no visible EdU uptake in control skin, while

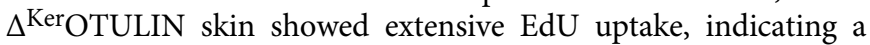
strong increase in epidermal stem cell proliferation (Fig. 2d). Imaging of tail wholemounts also revealed clear abnormalities in

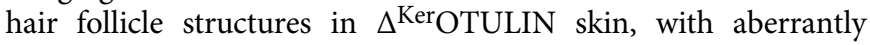
shaped sebaceous glands and marked thickening of the infundibulum (Fig. 2d). Immunofluorescent staining for cleaved caspase3 on tail wholemount sections confirmed the accumulation of caspase-3-positive dying cells over the entire length of $\Delta^{\text {Ker- }}$ OTULIN hair follicles, indicating that in absence of OTULIN, cell viability of hair follicle stem cell (HFSC) populations may be affected (Fig. 2e). To assess whether aberrant cell death precedes

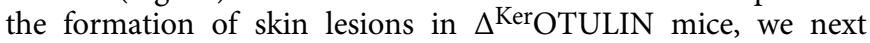
quantified the number of apoptotic cells in mice at a time-point when lesions were not yet apparent, namely at postnatal day P0.5. While epidermal thickness was not significantly altered yet in

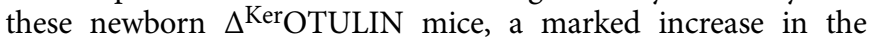
number of cleaved caspase-3-positive apoptotic interfollicular epidermis (IFE) cells could already be observed in the skin of these mice compared to control mice (Fig. $2 \mathrm{f}-\mathrm{h}$ ).

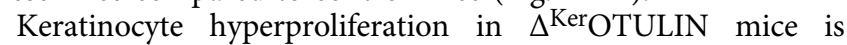
indicative for an increase in stem cell proliferative capacity, which is crucial for regenerative responses in the skin ${ }^{1}$. Indeed,

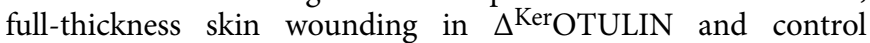
mice revealed a marked accelerated wound closure response in the initial phases of wound repair (day 2 and day 4 postwounding) in $\Delta^{\mathrm{Ker} O T U L I N}$ mice. However, when wounds enter the remodeling stage of repair (day 8 post-wounding), wound

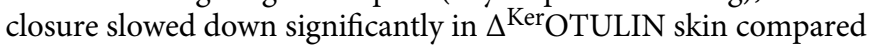
to control skin (Supplementary Fig. 2c-e). Intriguingly, $\Delta^{\mathrm{Ker} O T U L I N}$ skin developed cysts and tumor-like lesions at sites of wounding when re-epithelialization was complete
(Supplementary Fig. 2e), confirming the enhanced sensitivity of these mice to skin tumorigenesis.

TNFR1-mediated cell death drives inflammation in $\Delta^{\text {Ker- }}$ OTULIN mice. The cutaneous inflammation in Sharpin $c p d m / c p d m$ mice does not develop in the absence of TNFR $1^{5,30,31}$. However, the lethal dermatitis present in keratinocyte-specific HOIL-1 or HOIP deficient mice is only partially mediated by TNFR $1^{8}$. Therefore, we tested whether genetic ablation of TNFR1 also results in an amelioration of the inflammatory phenotype

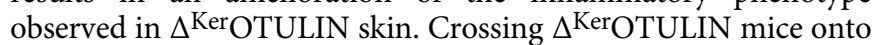
a TNFR1-deficient background completely prevented dermatitis (Fig. 3a), even at old age (Supplementary Fig. 3a), and $\Delta^{\text {Ker- }}$ OTULIN-TNFR $1^{-1-}$ mice showed significantly reduced IL6, TNF, and IL17 levels in their serum (Fig. 3b). Moreover, deletion of one functional TNFR1 allele partially protected $\Delta^{\text {KerOTULIN }}$ skin against the formation of skin lesions and inflammatory cytokine production (Fig. 3b). In agreement, the epidermis of

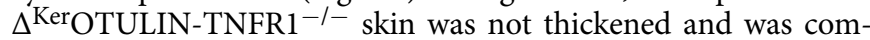
parable to the skin of control (OTULIN ${ }^{\mathrm{fl} / \mathrm{fl}}$ ) littermate mice (Fig. 3c).

Since RIPK1 kinase activity regulates cell death in the TNFR1complex II, we next evaluated the contribution of RIPK1 kinasedependent cell death to the inflammatory skin phenotype of

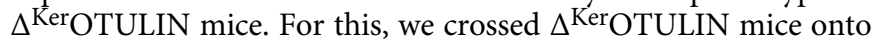
a RIPK1 kinase-inactive genetic (Ripk1 $\left.1^{D 138 N / D 138 N}\right)$ background.

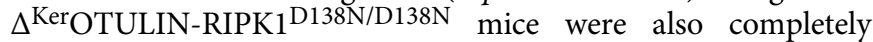
protected from dermatitis development (Fig. 3a), even at old age (40 weeks and older) (Supplementary Fig. 3b), and showed normal serum levels of inflammatory cytokines and chemokines (Fig. 3b), and normal thickness of the epidermis (Fig. 3c). RIPK1 kinase activity can induce both FADD-dependent apoptosis and RIPK3/MLKL-dependent necroptosis ${ }^{32}$. Hence, we next crossed $\Delta$ KerOTULIN mice with mice that have a floxed Fadd and Mlkl allele, generating mice that lack OTULIN, FADD, and MLKL specifically in keratinocytes. These $\Delta^{\mathrm{Ker} O T U L I N / F A D D / M L K L}$ mice were completely lesion-free (Fig. 3a and Supplementary Fig. 3c), exhibited normal circulating cytokine levels (Fig. 3b), and normal thickness of the epidermis (Fig. 3c), proving that cell

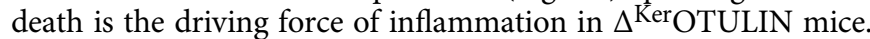
$\Delta$ Ker OTULIN mice only deficient for MLKL in keratinocytes were partially protected from dermatitis. The tail phenotype was

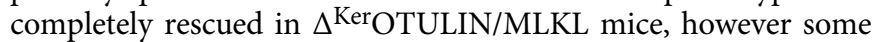
mice still developed lesions on the back skin (Supplementary Fig. 3d). Finally, we observed complete protection from

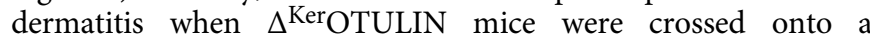
MyD88-deficient genetic background, suggesting that microbial 
a.

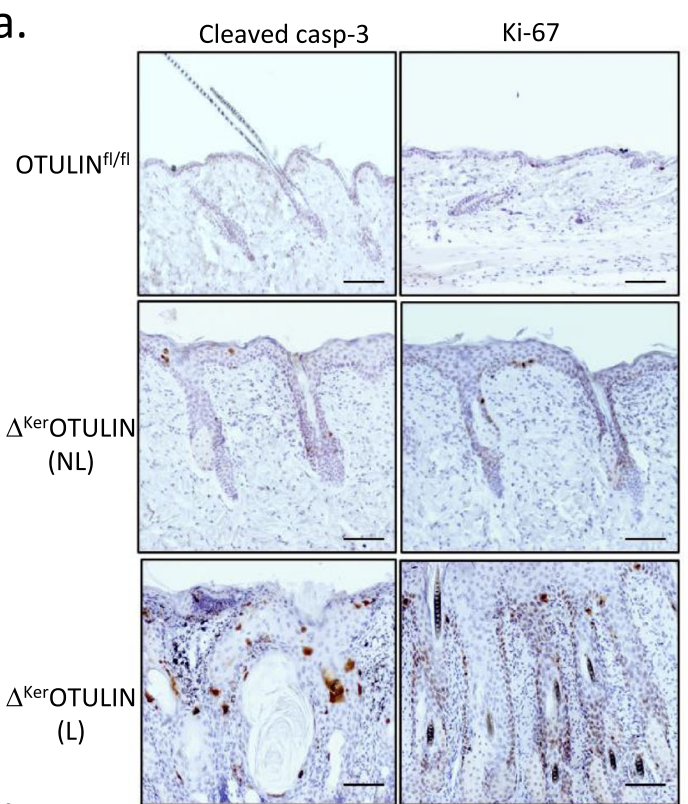

d.

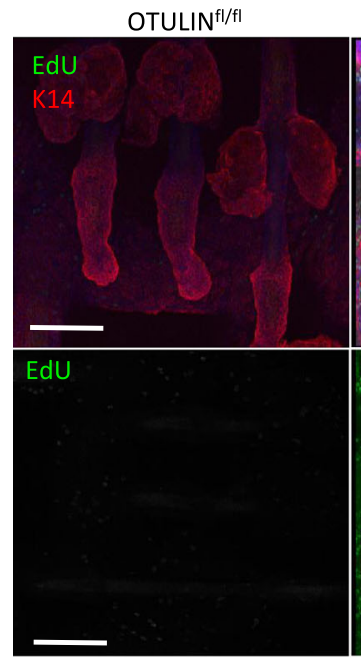

f.

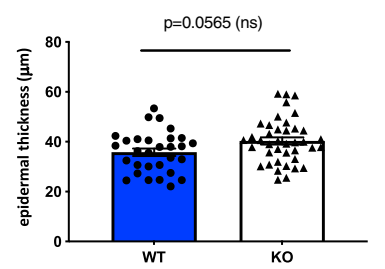

g.

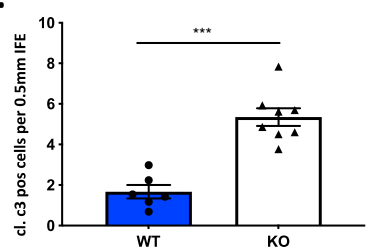

b.

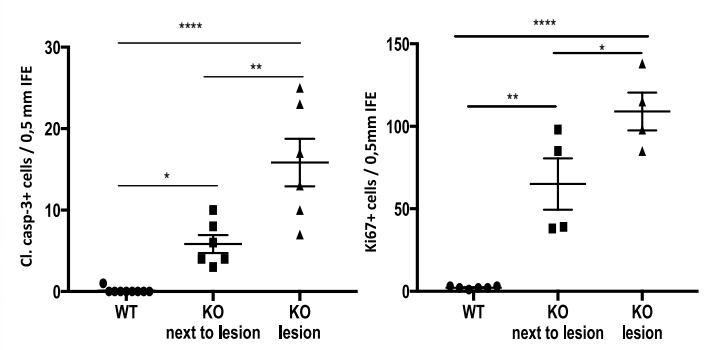

C.

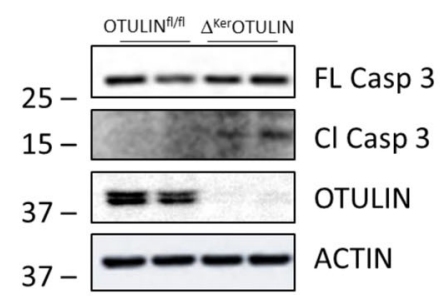

e.
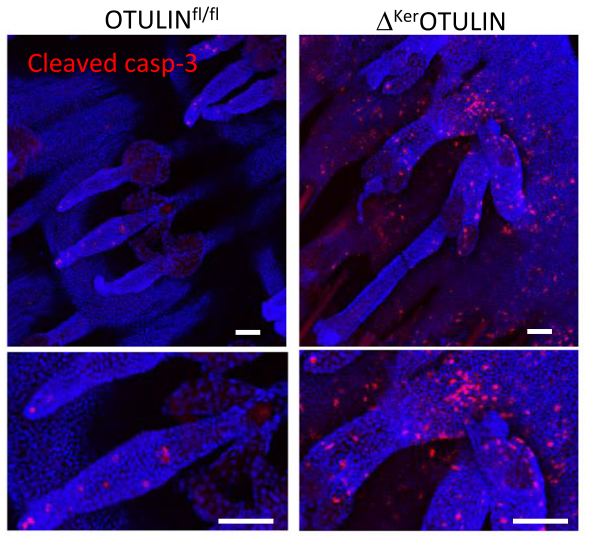

components could be involved in driving dermatitis in OTULINdeficient skin (Fig. 3a).

Primary mouse keratinocytes (PMKs) isolated from Shar

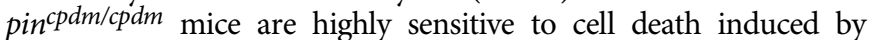
TNF stimulation ${ }^{6,33}$, and HOIP-deficient cells are less viable even in the absence of exogenous stimuli ${ }^{8}$. OTULIN-deficient PMKs, however, are equally resistant to TNF-induced cell death as control h.

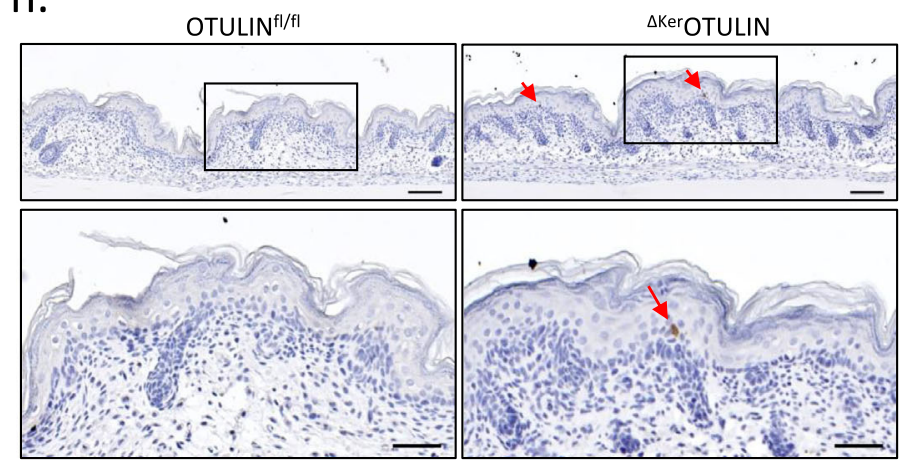




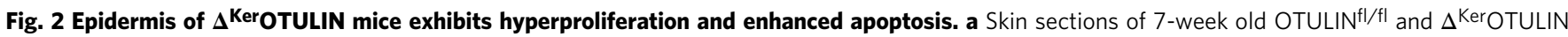
mice stained with antibodies against cleaved caspase-3 and Ki-67 to assess apoptosis and proliferation, respectively. NL non lesional; $L$ lesional. Scale bar:

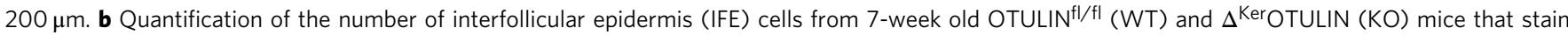
positive for cleaved caspase-3 and Ki-67 ( $n=9$ WT mice; $n=6$ and $4 \mathrm{KO}$ non-lesional mice per condition; $n=6$ and $4 \mathrm{KO}$ lesional mice; One-way ANOVA; $\left.{ }^{\star} p<0.5 ;{ }^{\star \star} p<0.01 ;{ }^{\star \star \star \star} p<0.0001\right)$. Data represent means $\pm \mathrm{SEM}$. c Western blot analysis for expression of full-length (FL) and cleaved (Cl) caspase-3 in epidermal tail lysates from OTULIN $\mathrm{N}^{\mathrm{f} / \mathrm{fl}}$ and $\Delta^{\mathrm{Ker} O T U L I N}$ mice. Anti-actin immunoblotting was used as a loading control. Molecular weight marker units are in kilodalton $(\mathrm{kD})$. This experiment was repeated three times independently with similar results. $\mathbf{d}$ EdU (green) retaining cells in tail

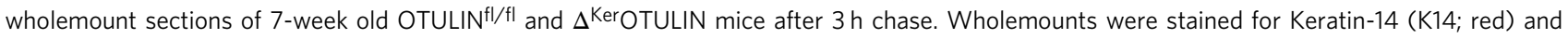
counterstained with Dapi (blue). Scale bars: $100 \mu \mathrm{m}$. Lower panels show EdU staining only. This experiment was repeated three times independently with

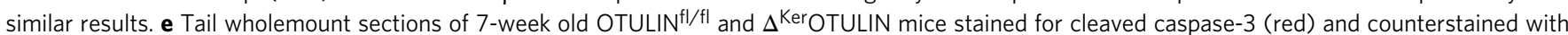
Dapi. Lower panels depict magnified views. Eight mice per genotype were analyzed. Scale bars: $50 \mu \mathrm{m}$. $\mathbf{f}, \mathbf{g}$ Quantification of epidermal thickness (f) and the

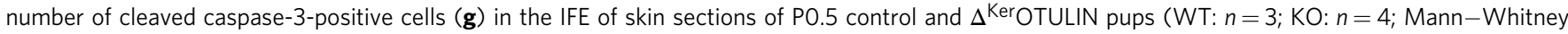
two-sided test; $\left.{ }^{\star \star \star} p=0.0007\right)$. Data represent means \pm SEM. h Representative images of cleaved capase-3-stained skin sections of OTULIN ${ }^{f l / f l}$ and

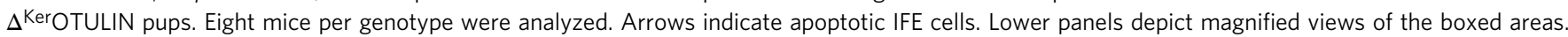
Scale bars: $100 \mu \mathrm{m}$.

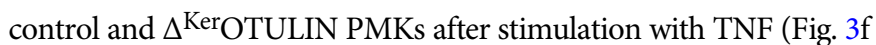
and Supplementary Fig. 3e). It should be noted that the residual

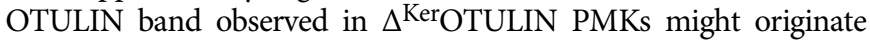
from feeder cells that can still be present in PMK cultures. A pronounced reduction could be observed in the expression of

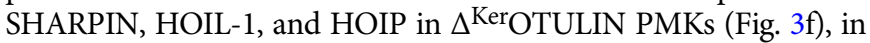
agreement with previous studies that have shown reduced expression of LUBAC components in OTULIN-deficient cells and tissues $15,17,24,28,29$, and consistent with the concept that OTULIN maintains LUBAC function by suppressing its auto-ubiquitination and degradation ${ }^{17}$. Indeed, pretreatment of PMKs with the proteasome inhibitor MG132 could restore SHARPIN and HOIP levels in $\Delta^{\mathrm{Ker} O T U L I N}$ cultures (Supplementary Fig. 3f). Finally, analysis of linear ubiquitination by specific pulldown of ubiquitinbinding domain-containing proteins using recombinant GSTUBAN (Ub-binding domain in ABIN proteins and NEMO), demonstrated a strong increase in M1-ubiquitination in primary OTULIN-deficient keratinocyte cultures after stimulation with TNF, compared to cultures from control mice (Fig. 3g), confirming the importance of OTULIN in restricting M1 ubiquitination in keratinocytes. Immunoblotting for RIPK1 in immunoprecipitation lysates of PMKs showed a decreased expression of RIPK1 in ${ }_{\Delta}$ KerOTULIN PMKs (Fig. 3g), although this altered RIPK1 expression was not observed in epidermal tails lysates isolated

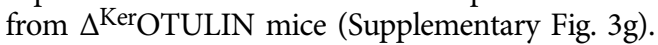

In conclusion, we could demonstrate that dermatitis and tumor

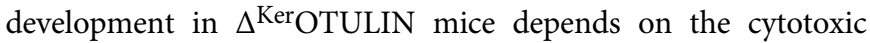
activity of TNF driving FADD- and RIPK1 kinase-dependent death of keratinocytes. The complete rescue from dermatitis upon genetic deletion of both FADD and MLKL proves that keratinocyte cell death is the driving force of the skin inflammation and tumorigenesis in $\Delta^{\mathrm{Ker}} \mathrm{OTULIN}$ mice.

OTULIN-deficiency in keratinocytes perturbs stem cell lineage and induces cutaneous infiltration of innate immune cells. To better characterize the inflammatory phenotype of $\Delta{ }^{\text {Ker OTULIN }}$ mice and to gain insights into the cellular differences between lesional and non-lesional $\Delta^{\mathrm{Ker} O T U L I N}$ skin, we next performed single-cell RNA-sequencing (scRNAseq) on live cells sorted from control wild-type (WT, OTULIN $\left.{ }^{\mathrm{fl} / \mathrm{fl}} ; n=1\right)$ skin and lesional (L;

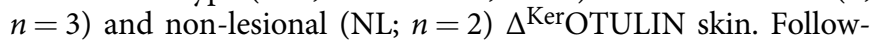
ing pre-processing of the data according to the Marioni pipeline, poor quality cells were excluded ${ }^{34}$. Firstly, unsupervised global clustering into populations was performed with affinity propagation according to the expression of high variance genes (Fig. 4a). The different cell populations that were delineated by unbiased clustering were annotated according to the expression of cell markers adapted from Joost et al. ${ }^{35}$ (Supplementary Fig. 4a).
Next, we determined which cells originated from the three different conditions. This analysis revealed a marked clustering of the control (WT) cells within the different cell populations, which was opposite to the distribution of the lesional cells (L) within the clusters. This was remarkably clear in the keratinocyte and

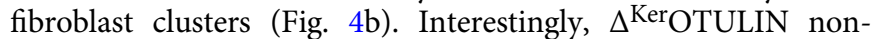
lesional (NL) cells were distributed over the entire keratinocyte and fibroblast cluster (Fig. $4 \mathrm{~b}$, middle panel). These cell-types are undergoing major changes in overall gene expression in non-

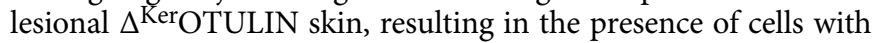
expression profiles that are highly similar to WT cells, alongside cells that are highly similar to lesional cells and cells that are clearly transitioning in between these two ends of the expression profile spectrum.

scRNAseq confirmed a strong infiltration of innate immune

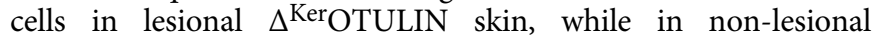

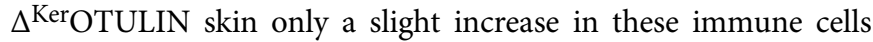
could be observed relative to the situation in WT skin (Fig. 4b, c). Subclustering and annotation of this immune cell population revealed that this cluster harbors mainly macrophages and dendritic cells (Fig. 4c). The gradual infiltration of immune cells

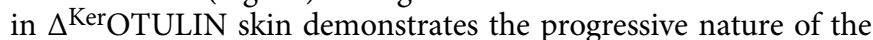
inflammatory phenotype that develops in these mice. The higher

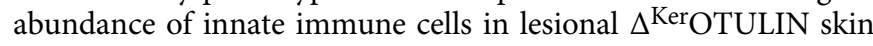
was confirmed by flow cytometry, showing a significant increase in the total number of CD45+ immune cells in lesional skin

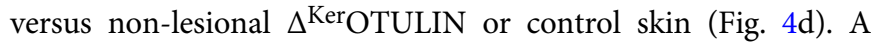
marked infiltration of F4/80-positive macrophages, cDC1, cDC2, eosinophils, and Langerhans cells was observed in lesional $\Delta$ KerOTULIN skin relative to non-lesional $\Delta^{\text {KerOTULIN } \text { or }}$ control skin in both flow cytometry and scRNAseq (Fig. 4d, e; Supplementary Fig. 4b). Interestingly, the most substantial

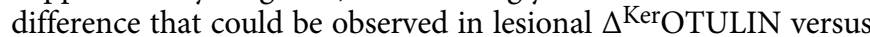
non-lesional skin according to flow cytometric analyses was an increase in the number of inflammatory macrophages (CD45+ $\mathrm{CD} 11 \mathrm{~b}+\mathrm{F} 4 / 80+$ cells) (Fig. 4d). scRNAseq analysis also revealed extensive changes in the T-cell population, where a substantial infiltration of regulatory T-cells (Tregs) occurred in both lesional

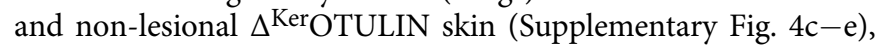
which was confirmed by flow cytometric quantification of the number of FoxP3 ${ }^{+}$T-cells (Supplementary Fig. 4f).

Our scRNAseq data also showed that several keratinocyte populations marked by HFSC markers, such as Lgr5+, Lrig1+, and Sox9+ HFSCs, show a gradual expansion in both lesional and non-lesional skin, while others, such as CD34+ keratinocytes, gradually decrease in frequency in non-lesional and lesional ${ }_{\Delta}$ KerOTULIN skin (Fig. 4f and Supplementary Fig. 4g). These data indicate that stem cells display a high degree of plasticity in 
a.
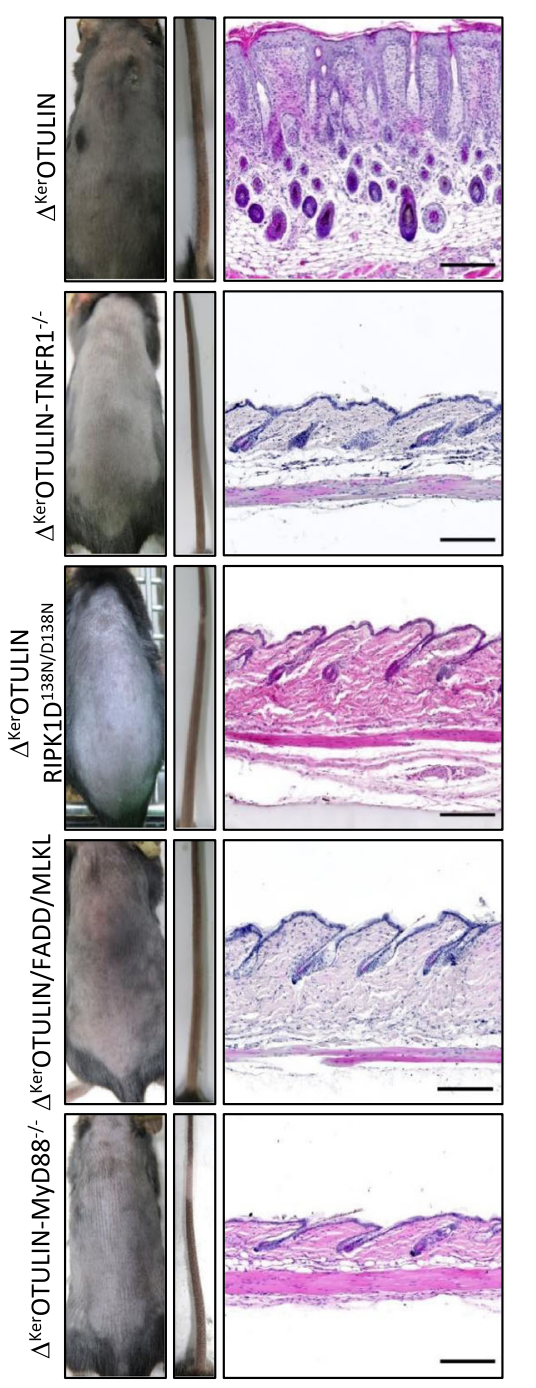

b.
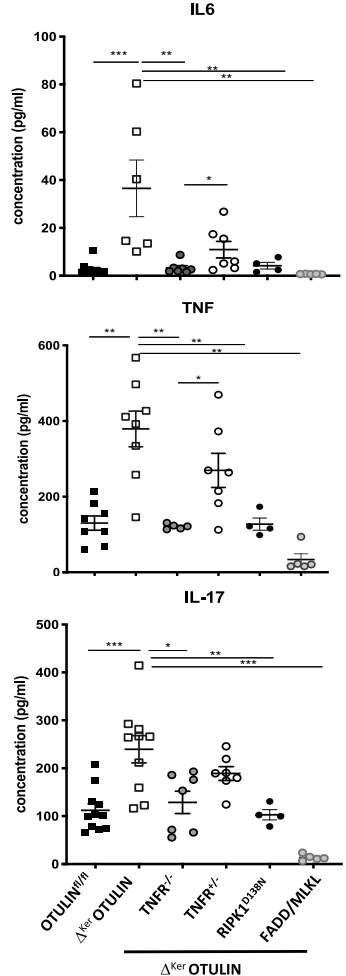

C.

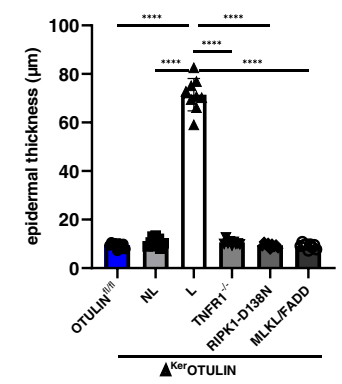

d.

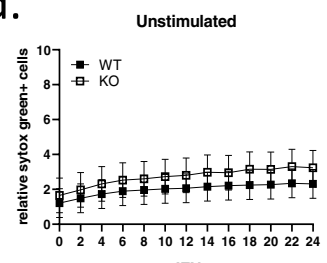

IFNY

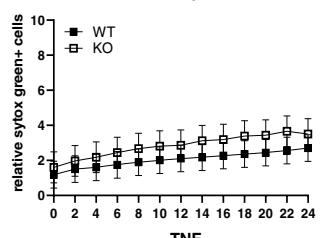

TNF

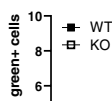

은.

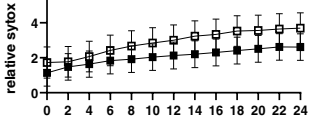

IFNY + TNF

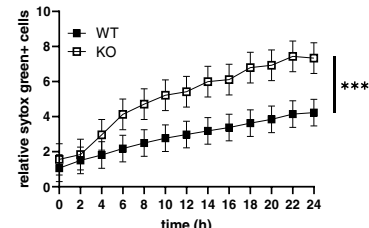

e.

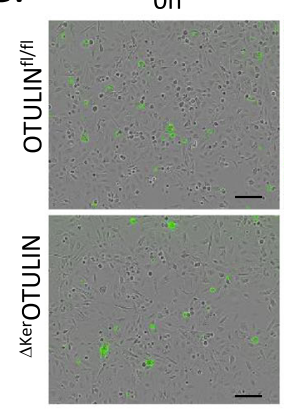
$24 \mathrm{~h}$

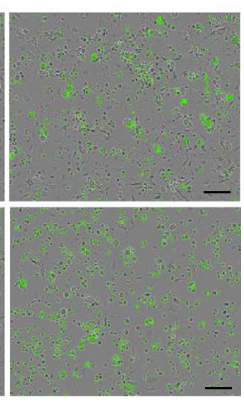

f.

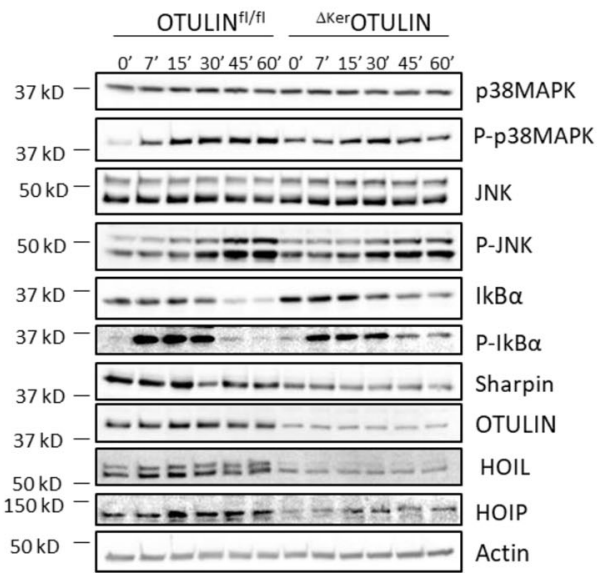

g.

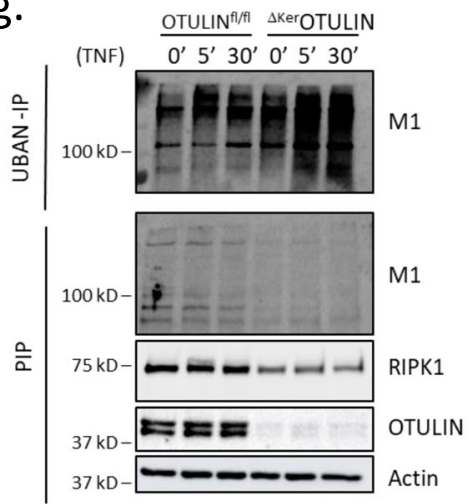

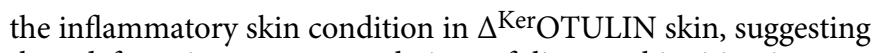
that defects in proper regulation of linear ubiquitination are important for stem cell lineage in the skin. These stem cell changes are already initiated prior to the massive infiltration of immune cells into the skin, as stem cell populations exhibit

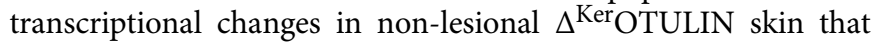

does not harbor significantly more immune cells than control skin (Fig. 4e, f).

Type-1 interferons contribute to dermatitis in $\Delta^{\text {Ker OTULIN }}$ mice. Recent studies identified an important role for type-1 


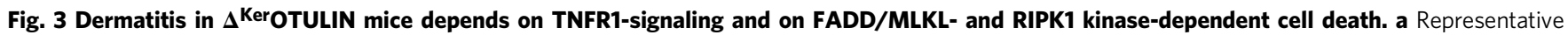
images of the back skin and tail of 7-week old mice of the indicated genotype. The right panel shows H\&E-stained skin sections of the respective mice. Scale

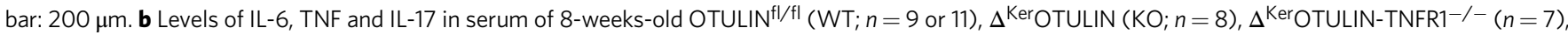

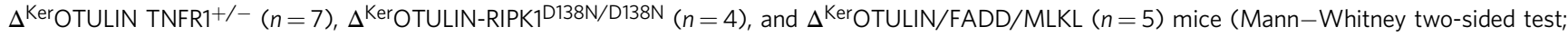
$\left.{ }^{\star} p<0.5 ;{ }^{\star \star} p<0.01 ;{ }^{\star \star \star} p<0.001 ;{ }^{\star \star \star \star} p<0.0001\right)$. Data represent means \pm SEM. c Epidermal thickness quantification at 7-11 weeks of age. NL non-lesional; $L$ lesional ( $n=10$ per condition; ${ }^{\star \star \star \star} p<0.0001$, One-way ANOVA with multiple comparisons). Data represent means \pm SEM. d Primary keratinocyte cultures ( $n=3$ biological replicates per condition) were treated with $20 \mathrm{ng} / \mathrm{ml} \mathrm{mTNF}$ with or without priming with IFN- $\gamma$ (10 ng/ml) $8 \mathrm{~h}$ prior to TNF stimulation. Viability was assessed by Sytox Green uptake. Representative graphs for three independent experiments. (Residual maximum likelihood (REML);

$\left.{ }^{\star \star *} p=0.002\right)$. Data represent means \pm SEM. e Incucyte images depicting Sytox Green uptake by dead keratinocytes at 0 and $24 \mathrm{~h}$ post IFN- $\gamma$ and TNF treatment. This experiment was repeated three times independently with similar results. $\mathbf{f}$ Western blot analysis on lysates from primary keratinocyte cultures

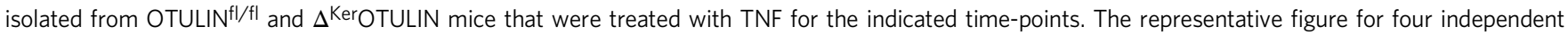

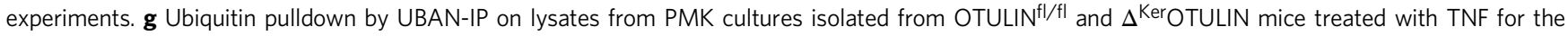
indicated time-points followed by immunoblotting for M1 Ubq chains. Pre-immunoprecipitation lysate (PIP) was immunoblotted for M1 Ubq (M1), RIPK1, and OTULIN. $\alpha$-Actin is shown as loading control. This experiment was repeated three times independently with similar results.

interferons (IFNs) in the process of inflammation ${ }^{4,17,24}$. Expression analysis of IFN-stimulated genes (ISGs) using our scRNAseq dataset indeed revealed an increased expression of ISGs in OTULIN-deficient keratinocytes. Remarkably, multiple ISGs such as $\operatorname{Irf3}$, Irf9, and USP18 were already upregulated in non-lesional ${ }_{\Delta}$ KerOTULIN skin, implicating that IFN signaling is an early event in the generation of dermatitic lesions (Fig. 5a). Q-PCR analysis of $\Delta^{\mathrm{Ker} O T U L I N}$ and control epidermal tail lysates confirmed the upregulation of ISGs and type-1 IFNs in keratinocytes in the absence of OTULIN (Fig. 5b).

To further investigate whether type-1 IFNs are crucial in

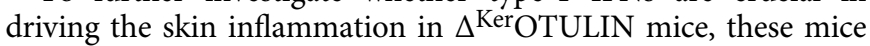
were crossed to Ifnar1 (Interferon-a receptor 1)-deficient mice. $\Delta^{\text {KerOTULIN-IFNAR1 }}{ }^{-l-}$ mice showed a rescue of the skin phenotype with lower lesion incidence and skin lesions develop-

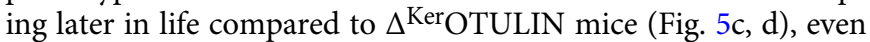
at old age ( $>40$ weeks of age) (Supplementary Fig. $3 \mathrm{~h}$ ). In agreement, serum levels of IL-6, TNF, IL-17, and MCP-1 were

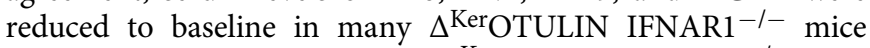

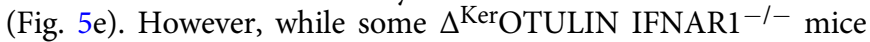
were completely protected from dermatitis, others still developed skin inflammation over time (Fig. $5 \mathrm{~d}-\mathrm{f}$ ). Finally, immunostaining for the type- 1 interferon IFN- $\beta$ revealed marked expression of

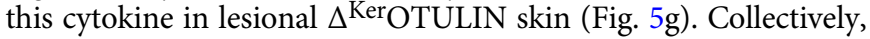
these data demonstrate that the production of type-1 IFNs critically contributes to the formation of inflammatory skin lesions in $\Delta^{\text {Ker OTULIN mice. }}$

Dermatitis in $\Delta^{\text {Ker }}$ OTULIN mice is mediated by Interleukin-1 $\beta$ released by innate immune cells. We next set out to identify the signals driving the response of OTULIN-deficient keratinocytes to inflammation. Therefore, we made use of the NicheNet algorithm designed to infer ligand-receptor links between interacting cells by combining transcriptome data of interacting cells with existing knowledge on gene regulatory networks ${ }^{36}$. NicheNet analysis was applied to predict the ligands that are produced by innate immune cells and bind to receptors on keratinocytes, causing the changes in keratinocyte gene expression profiles (Fig. 6a). One of the top predicted ligands which we identified by NicheNet to be produced by infiltrating innate immune cells in lesional skin of $\Delta^{\text {Ker- }}$ OTULIN mice and modulating gene expression in keratinocytes, was the cytokine IL-1 $\beta$ (Fig. 6a). This cytokine was also identified by NicheNet as a ligand with putative regulatory potential in keratinocytes when comparing non-lesional skin to control skin (Supplementary Fig. 5a), indicating that IL-1 $\beta$ could be an early mediator of the aberrant keratinocyte behavior in $\Delta^{\text {KerOTULIN }}$ skin. We next assessed the expression profile of IL- $1 \beta$ and the IL-1 family members IL- $1 \alpha$ and IL-18 in our scRNAseq dataset and could observe that IL-1 $\beta$ was indeed strongly produced by immune cells infiltrating lesional skin of $\Delta^{\text {KerOTULIN mice }}$ (Fig. 6b and Supplementary Fig. 5b). This expression profiling also revealed that macrophages represent the predominant IL-1 $\beta$ producing cell population (Fig. 6b). Interestingly, next to IL-1 $\beta$ and IL-18, also other genes involved in inflammasome activation and IL- $1 \beta$ production, including caspase-1, ASC, and Nlrp3, were upregulated in keratinocytes of lesional skin of $\Delta^{\text {Ker }}$ OTULIN mice

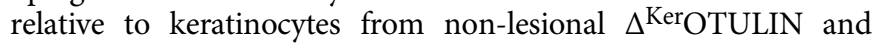
wild-type mice (Fig. 6c). To assess the functional importance of IL-1 $\beta$ in the pathology of $\Delta^{\mathrm{Ker} O T U L I N}$ mice, we next treated $\Delta$ KerOTULIN mice with Anakinra, a recombinant version of the human interleukin-1 receptor (IL1R), blocking the binding of IL$1 \alpha$ and IL- $1 \beta$ to the IL1R ${ }^{37}$. Daily intraperitoneal injections of

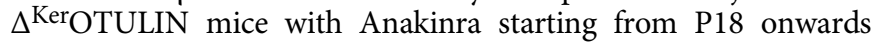
could suppress dermatitis development in the back skin and tail of these mice, confirming an important contribution of IL- $1 \beta$ in the development of skin lesions (Fig. 6d and Supplementary Fig. 6). The therapeutic potency of Anakinra treatment to ameliorate

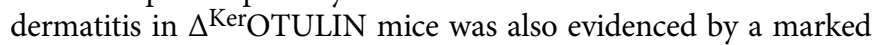
restoration of the epidermal permeability function in the lesional skin of these mice (Fig. 6e). These data demonstrate that IL-1 $\beta$ production by infiltrating immune cells contributes to the inflammatory skin phenotype in $\Delta^{\text {KerOTULIN mice. }}$

Next to the cytokine IL- $1 \beta$, we also identified the chemokine MCP-1 by NicheNet as a ligand that could mediate transcriptional changes in keratinocytes when comparing non-lesional skin to control skin (Supplementary Fig. 5a). MCP-1 is a potent chemokine attracting macrophages, therefore we investigated whether blocking MCP-1 could suppress dermatitis in $\Delta^{\mathrm{Ker}} \mathrm{O}$ TULIN mice. Indeed, intraperitoneal injections of $\Delta{ }^{\text {Ker OTULIN }}$ mice with a neutralizing $\alpha-\mathrm{MCP}-1$ antibody could ameliorate but not fully suppress the dermatitis in back skin, and completely

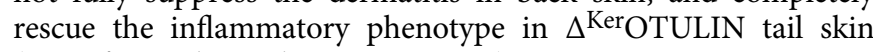
(Fig. 6f, g and Supplementary Fig. 6).

Knockin of a human mutation in the murine Otulin gene phenocopies OTULIN-deficiency. Homozygous hypomorphic mutations in the human OTULIN gene, affecting the deubiquitinase activity of the protein, have been shown to underlie the development of a severe life-threatening autoinflammatory syndrome, called ORAS ${ }^{15,16}$. ORAS patients develop neonatal-onset fever, swollen joints, and diarrhea, but also dermatitis and panniculitis ${ }^{15,16,38}$. The best characterized homozygous missense mutation L272P (c.815T > C;p Leu272Pro) was shown to result in reduced OTULIN stability and activity towards M1 linked ubiquitin, and patients' fibroblasts and peripheral blood mononuclear cells showed evidence for increased NF- $\kappa B$ signaling and production of inflammatory cytokines ${ }^{15,16}$. 
a.

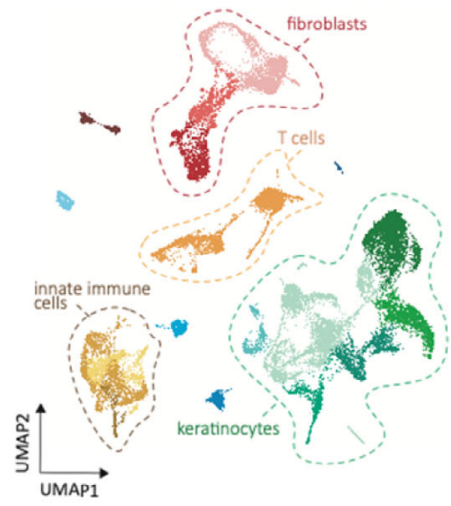

c.

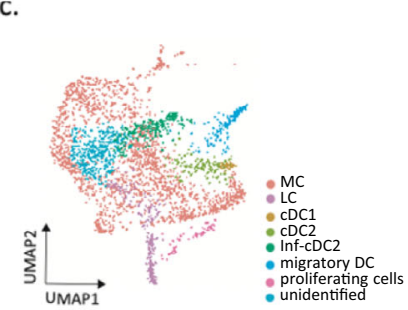

b.

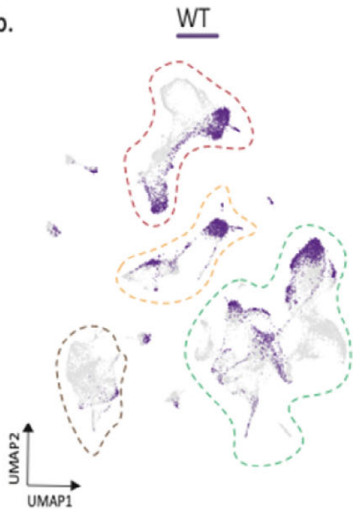

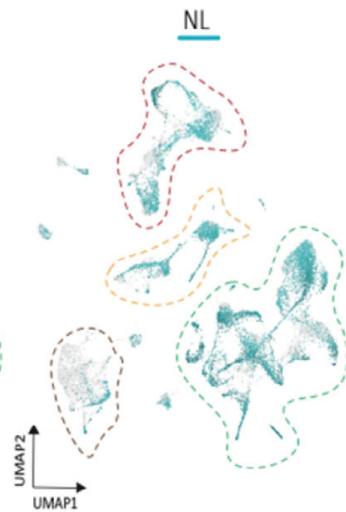

d.

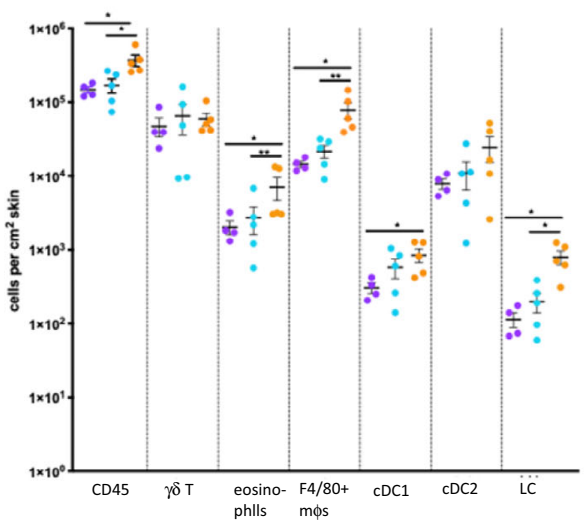

WT

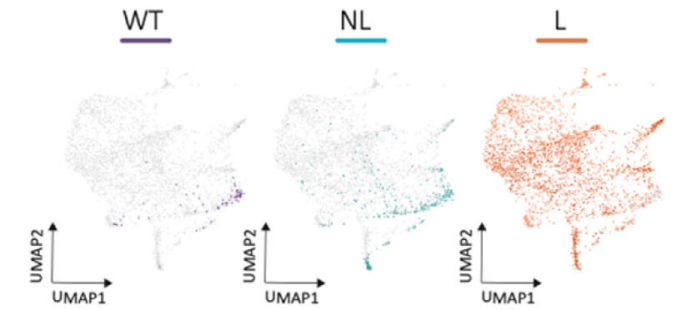

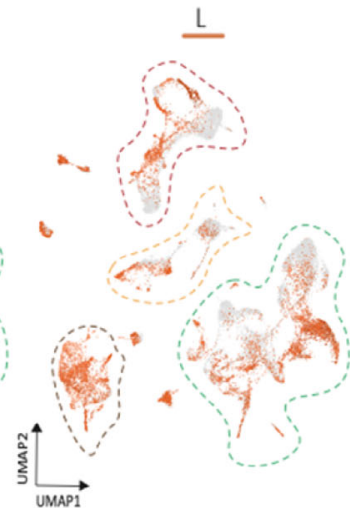

e.

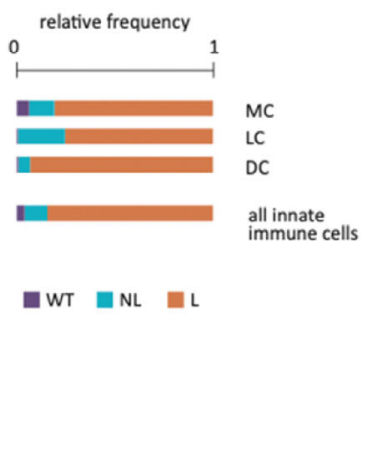

f.

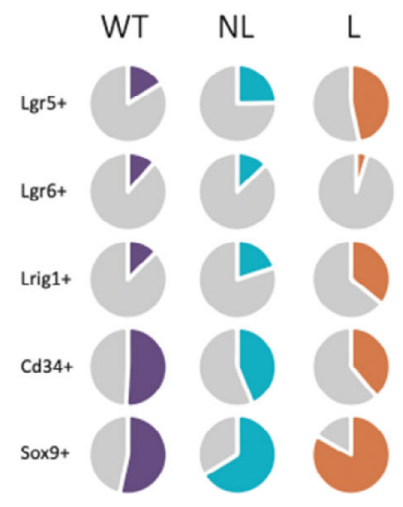

Fig. 4 Single-cell RNA-sequencing reveals marked infiltration of innate immune cells and changes in hair follicle stem cell lineage in $\Delta^{\text {KerOTULIN }}$ mice. a Annotated UMAP clustering of live skin cells isolated from control OTULINfl/fl mice (WT) and from non-lesional (NL) and lesional (L) total skin of

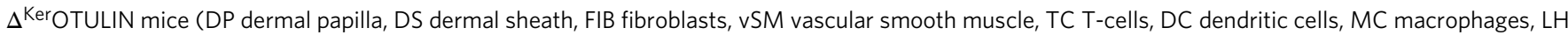
Langerhans cells, IFE interfollicular epidermis (including infundibulum), HF IL hair follicle anagen inner layer cells, SG sebaceous gland, uHF upper hair follicle, HF OL hair follicle anagen outer layer cells, HF Bu hair follicle bulge, EC endothelial cells, LV lymph vessel, MEL melanocytes, SC Schwann cells). b Distribution of WT, NL, and L cells within the cell clusters. c UMAP plot of annotated innate immune cell subcluster, showing assigned clusters and distribution of WT, NL, and L cells within this cluster. $\mathbf{d}$ Flow cytometric analysis of immune cell composition in control OTULIN $\mathrm{Nl}^{\mathrm{fl}}$ skin $(\mathrm{WT}, n=4$ mice)

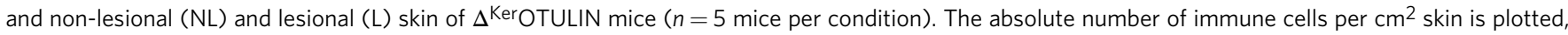
data represent means \pm SEM. (Mann-Whitney two-sided test; ${ }^{\star} p<0.5 ;{ }^{\star \star} p<0.01 ;{ }^{\star \star \star} p<0.001 ;{ }^{\star \star \star \star} p<0.0001$ ). e Relative normalized cell frequencies of the indicated immune cell-types in the different conditions ( $\mathrm{LH}$ Langerhans cells, MC macrophages, DC dendritic cells). $\mathbf{f}$ percentage of keratinocytes expressing the indicated HFSC markers within the total keratinocyte population of WT, NL and L skin.

To experimentally assess the importance of the L272P mutation, we generated a novel knockin transgenic mouse line expressing the OTULIN ${ }^{\mathrm{L} 272 \mathrm{P}}$ mutation (amino acid L272 being conserved in mouse) through CRISPR/Cas gene-editing technology. The desired point mutation was verified by PCR amplification and sequencing of the DNA sequence around the target sites (Fig. 7a). Heterozygous OTULIN ${ }^{\mathrm{L} 272 \mathrm{P} /+}$ knockin mice were crossed to homozygosity, but no homozygous OTULIN $^{\mathrm{L} 272 \mathrm{P} / \mathrm{L} 272 \mathrm{P}}$ mice were born (Table 1), confirming the lethal phenotype caused by the loss of the deubiquitinase function in these mice, in agreement with what has been shown before in OTULIN knockout mice ${ }^{15,24}$, in knockin mice that express catalytically inactive (C129A) OTULIN ${ }^{17}$, and in gumby mice that have a point mutation (W96R or D336E) in Otulin that abolishes its ability to bind to ubiquitin ${ }^{13}$. However, the OTULIN ${ }^{\mathrm{L} 272 \mathrm{P} / \mathrm{L} 272 \mathrm{P}}$ lethality could be rescued when OTULIN $^{\mathrm{L} 272 \mathrm{P}}$ mice were crossed into a caspase- 8 and RIPK3 deficient background (Table 1 and Fig. 7b, c). OTULIN ${ }^{\mathrm{L} 272 \mathrm{P} /}$ ${ }^{2} 272 \mathrm{P}_{\text {casp }} 8^{-/-} \mathrm{RIPK}^{-/-}$mice were born in Mendelian numbers and developed normally without any sign of inflammation (Table 1, Fig. 7b, c) with the exception of the lymphoproliferative syndrome that develops in Casp $8^{-1-}$ RIPK3 ${ }^{-1-}$ mice, as previously shown ${ }^{39,40}$. This rescue implies that aberrant cell death triggers lethality in OTULIN ${ }^{\mathrm{L} 272 \mathrm{P} / \mathrm{L} 272 \mathrm{P}}$ mice. 
a.

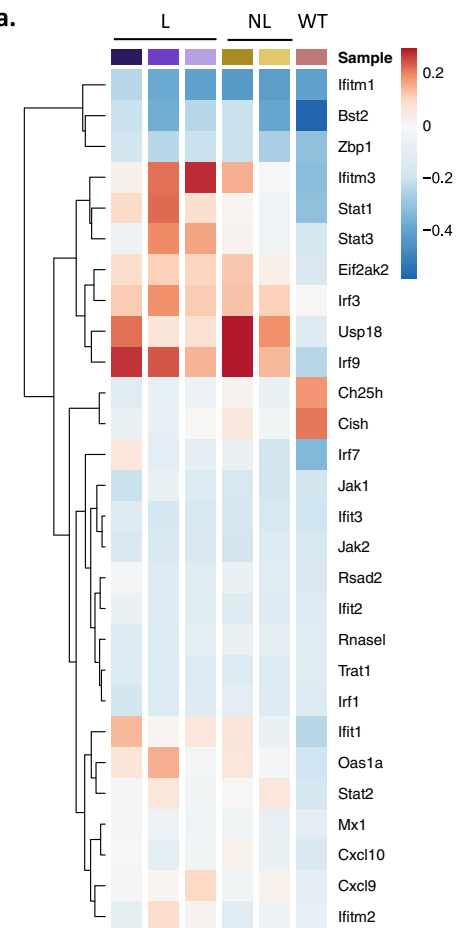

c.

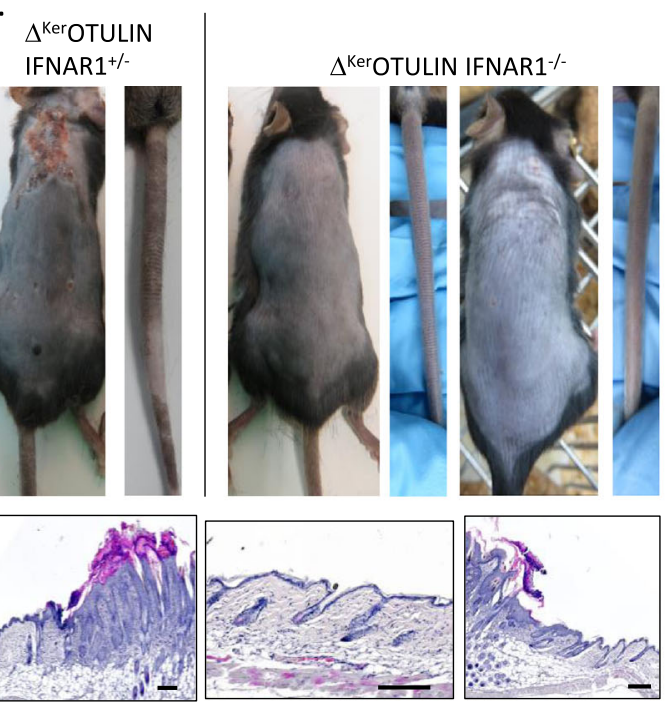

f.

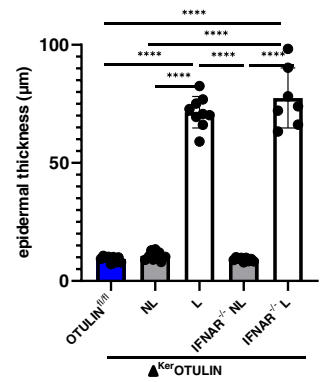

b.

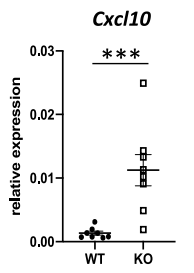

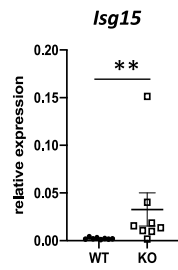

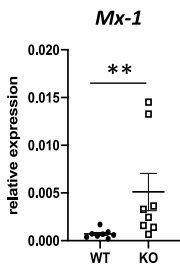
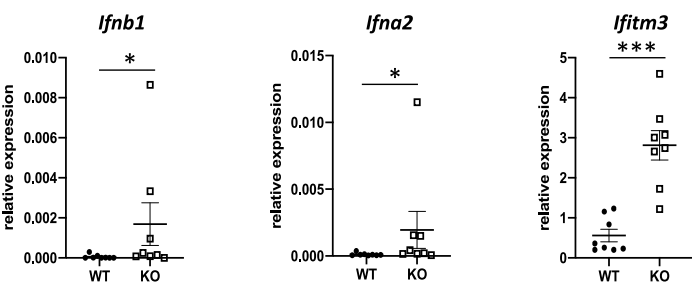

d.
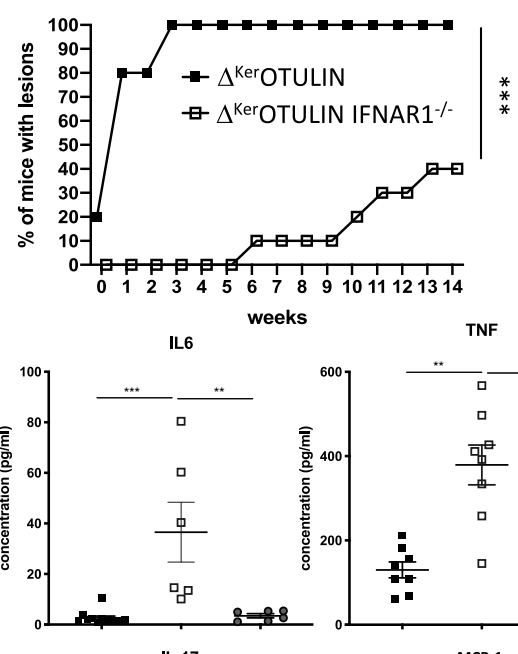

TNF
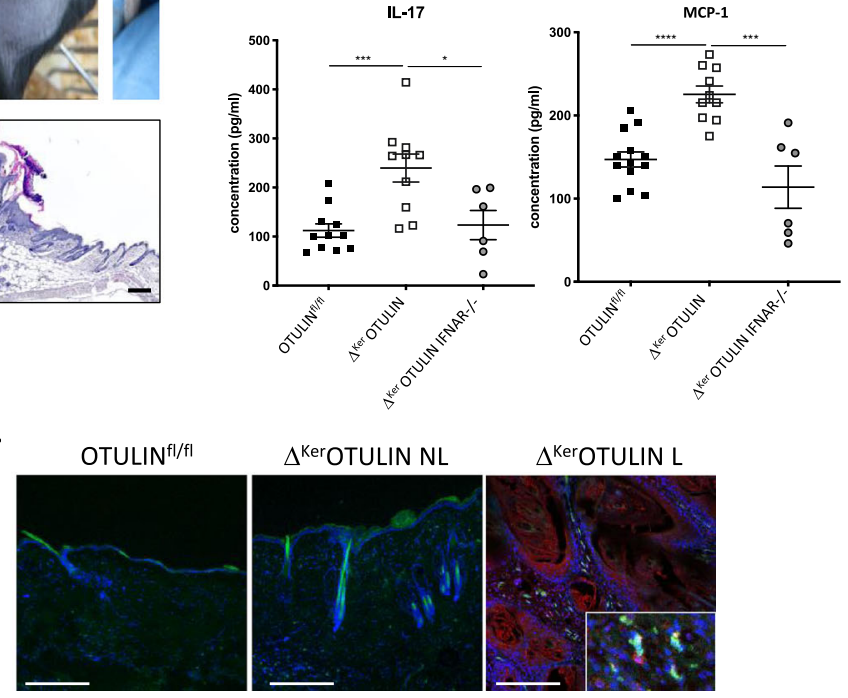

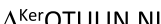

$\Delta^{\text {KerOTULIN L }}$

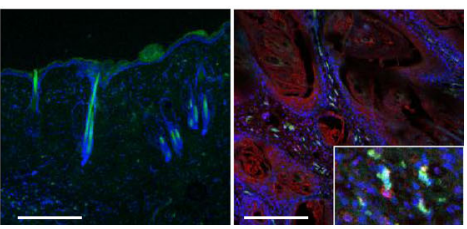

We next crossed OTULIN ${ }^{\mathrm{L} 272 \mathrm{P} /+}$ knockin mice with heterozygous keratinocyte-specific OTULIN deficient mice, generating OTULIN $^{\mathrm{L} 272 \mathrm{P} / \Delta \mathrm{Ker}}$ mice, having one L272P knockin allele in all cells and tissues, and one OTULIN knockout allele specifically in keratinocytes. These mice develop skin lesions and verrucous carcinomas on their back skin in a similar manner and timeframe

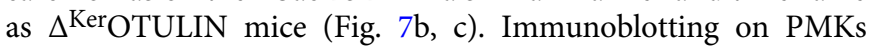

isolated from OTULIN ${ }^{\mathrm{L} 272 \mathrm{P} / \Delta \mathrm{Ker}}$ mice confirmed the reduced stability of OTULIN, SHARPIN, and HOIP (Fig. 7d).

Together, these findings demonstrate that expression of a humanrelevant OTULIN mutation in mice induces a similar inflammatory skin phenotype as observed in keratinocyte-specific OTULIN knockout mice, confirming that proper regulation of linear protein ubiquitination is crucial for mammalian skin homeostasis. 


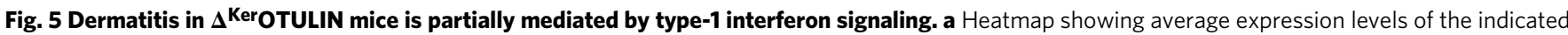
interferon-response genes in keratinocytes of the different conditions as observed in single-cell RNA-seq data. b Relative mRNA expression of Cxcl-10,

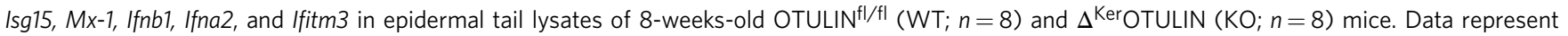
means \pm SEM. $\left({ }^{\star} p<0.05 ;{ }^{\star \star} p<0.01 ;{ }^{\star \star \star} p<0.001\right.$; Mann-Whitney two-sided test). c Representative images of the back skin and tail (upper panel), and

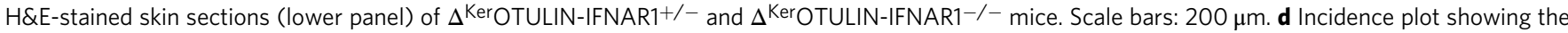

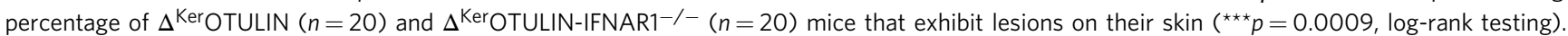

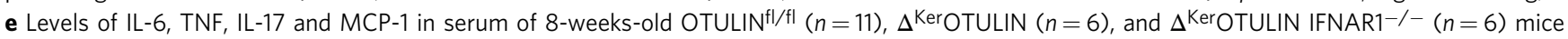
(Mann-Whitney two-sided test; ${ }^{\star} p<0.5 ;{ }^{\star \star} p<0.01 ;{ }^{\star \star \star} p<0.001 ;{ }^{\star \star \star \star} p<0.0001$ ). Data represent means \pm SEM. f Epidermal thickness quantification of skin of 7-11 weeks old mice. NL non-lesional; L lesional ( $n=8$ or 10 per condition; ${ }^{\star \star \star \star} p<0.0001$, One-way ANOVA with multiple comparisons). Data represent means \pm SEM. $\mathbf{g}$ Immunofluorescent staining for CD45 (green) and IFN- $\beta$ (red) on skin sections from OTULIN ${ }^{\mathrm{fl} / \mathrm{fl}}$ mice and non-lesional (NL) and

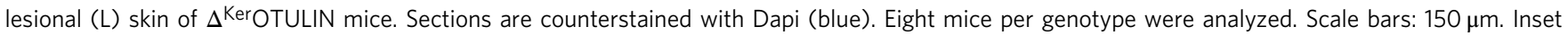
depicts a magnified view.

\section{Discussion}

Functional deletion of single LUBAC components leads to the development of inflammatory skin phenotypes of varying severity. While the skin of Sharpin ${ }^{c p d m / c p d m}$ mice exhibits overall inflammation resembling atopic dermatitis, mice that lack HOIP or HOIL-1 selectively in keratinocytes develop a more severe cutaneous inflammation that results in early postnatal lethality $8,10,30,31$. Here, we show that mice lacking OTULIN selectively in keratinocytes exhibit a severe skin inflammation that presents on their tail skin and delineated regions of the back skin. These inflammatory lesions develop into verrucous carcinomas, an uncommon variant of squamous cell carcinomas, that are characterized by exophytic epidermal outgrowths and marked melanophagy, a phenotype that was not observed in mice lacking components of the LUBAC complex. Also, the degree of cuta-

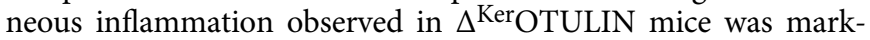
edly milder than the severe skin phenotype observed in mice lacking HOIP or HOIL-1 in keratinocytes, which leads to early postnatal lethality ${ }^{8}$. Although $\Delta^{\text {Ker OTULIN skin and keratino- }}$ cytes showed a significant reduction in expression of LUBAC proteins, still residual LUBAC activity in the skin of these mice can be expected.

$\Delta^{\mathrm{Ker} O T U L I N}$ mice are fully protected from dermatitis and skin tumorigenesis when crossed to a TNFR1-deficient or RIPK1 kinase-mutant background. This is in agreement with the phenotype in Sharpincpdm/cpdm mice that also do not develop skin inflammation in the absence of TNFR1 or RIPK1 kinase signaling ${ }^{30,31}$, but is in contrast to the phenotype of keratinocytespecific HOIP or HOIL-1 knockout mice that only show a delayed dermatitis in TNFR1 deficient or RIPK1 kinase-dead conditions ${ }^{8}$. Also, in contrast to primary keratinocytes from Sharpin ${ }^{c p d m / c p d m}$ mice that are highly sensitive to TNF-induced cell death, OTULIN-deficient keratinocytes are equally resistant to TNF-induced cell death as control keratinocytes. However, when primary keratinocyte cultures were primed with IFN- $\gamma$,

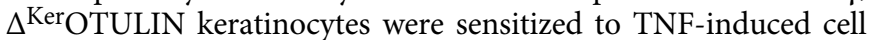
death. No differences, however, could be observed in TNFinduced NF- $\kappa \mathrm{B}$ and MAPK responses between control and

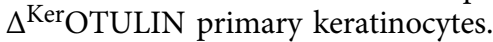

The observation that genetic deletion of both FADD and

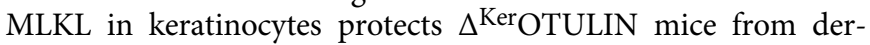
matitis development, proved that cell death of keratinocytes is the driving force of the cutaneous inflammation developing in $\Delta^{\mathrm{Ker} O T U L I N}$ mice. MLKL deficiency could ameliorate but not

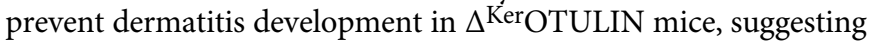
that both FADD-dependent apoptosis and MLKL-dependent necroptosis are driving the skin lesion development in $\Delta^{\text {Ker- }}$ OTULIN mice. Moreover, our data point to keratinocyte cell death preceding inflammation, as we observed apoptotic keratinocytes in $\Delta^{\mathrm{Ker} O T U L I N}$ skin sections at a time-point (P0.5) when skin lesions were not apparent yet. Also, the absolute number of infiltrating immune cells was still largely comparable

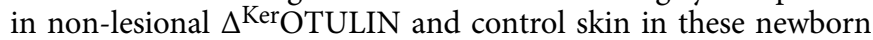
mice, and no increase in epidermal thickness could be observed, again suggesting that keratinocyte death occurs prior to the inflammation. Our scRNAseq data also pointed out that several subsets of HFSCs expand in a progressive manner in non-lesional

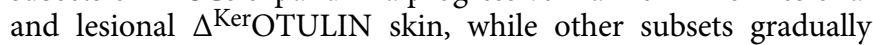
decrease in numbers. Whether these changes in stem cell lineage are due to changes in stem cell death rates or changes in proliferation under influence of OTULIN-deficiency, remains to be elucidated. This withstanding, it is clear that changes in keratinocyte stem cell fates occur prior to the substantial immune infiltration that is present in $\Delta^{\text {Ker }}$ OTULIN skin lesions.

We were able to identify an important role for type 1 IFN signaling in mediating skin inflammation in $\Delta^{\mathrm{Ker} O T U L I N}$ mice. Indeed, IFNAR1 deficiency rescued $\Delta^{\mathrm{Ker} O T U L I N}$ mice from dermatitis development in about $60 \%$ of the mice. These findings agree with previous studies pinpointing a role for type-1 IFNs in OTULIN deficient or mutant mice $4,17,24$, but implicate that other cytokines are also involved in regulating cutaneous inflammation in $\Delta^{\mathrm{Ker} O T U L I N}$ skin. However, it should be noted that the IFNAR1 knockout mice have various immune defects ${ }^{41}$, and were shown to be resistant to imiquimod-induced skin inflammation ${ }^{42,43}$. Prediction of ligandtarget cell interactions, by combining single-cell expression data with prior knowledge on signaling and gene regulatory networks, allowed us to also identify IL1 $\beta$ as an important cytokine involved in the

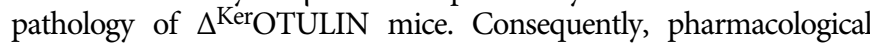
inhibition of IL1 $\beta$ signaling suppressed dermatitis development in

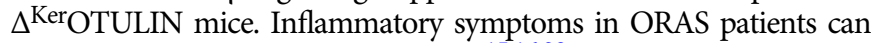
be managed by neutralization of TNF ${ }^{15,16,29}$, but IL1 $\beta$ neutralization by Anakinra showed efficacy in a patient with panniculitis and dermatosis ${ }^{16}$, in agreement with our findings in mice.

OTULIN deficiency has distinct biological effects in different cellular and tissue contexts. OTULIN deletion in macrophages was shown to induce systemic inflammation in mice, while deletion of OTULIN in B- or T-cells did not result in overt phenotypes $^{15}$. We and others recently showed that selective ablation of OTULIN in hepatocytes (OTULIN ${ }^{\mathrm{LPC}-\mathrm{KO}}$ ) results in severe liver disease characterized by fibrotic and neoplastic responses $^{24,28}$. While FADD deficiency or RIPK1 kinase-dead expression prevented hepatocyte cell death and development of liver disease in OTULIN ${ }^{\mathrm{LPC}-\mathrm{KO}}$ mice, genetic deletion of TNF or TNFR1 did not ameliorate the liver phenotype ${ }^{24}$, in sharp contrast with our observations in keratinocyte-specific OTULINdeficient mice, where ablation of TNFR1 signaling resulted in a complete rescue of cutaneous inflammation. These data indicate that OTULIN regulates inflammatory cell death pathways with different modes of action, depending on the cellular context. One possible explanation for this is the difference in Otulin expression levels across different tissues. Indeed, analysis of gene expression 
a.

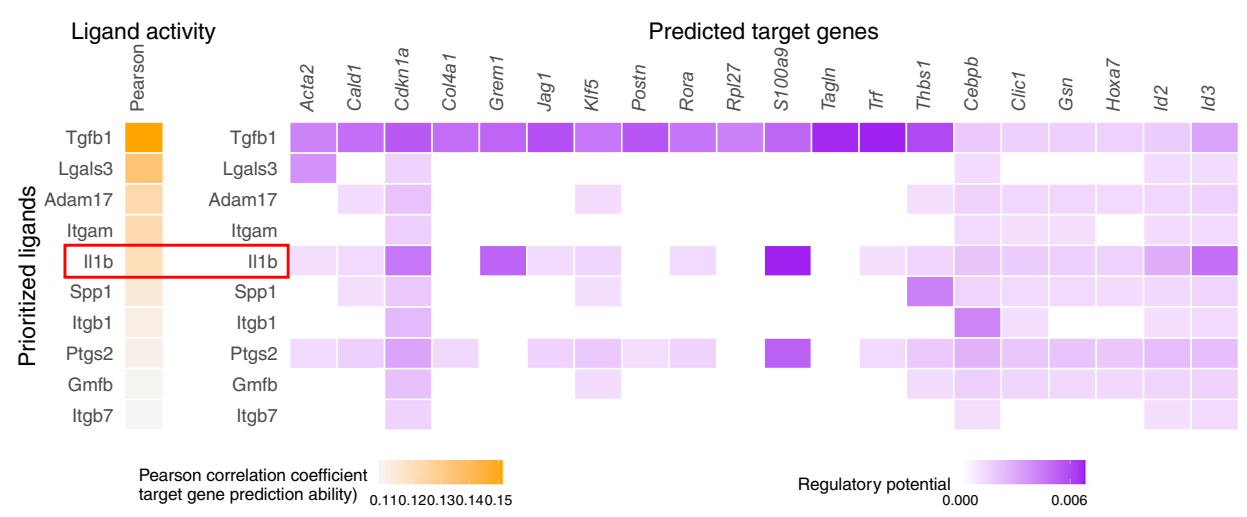

b.

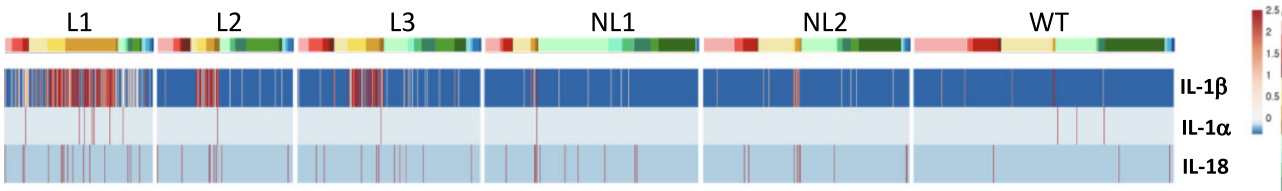

c.

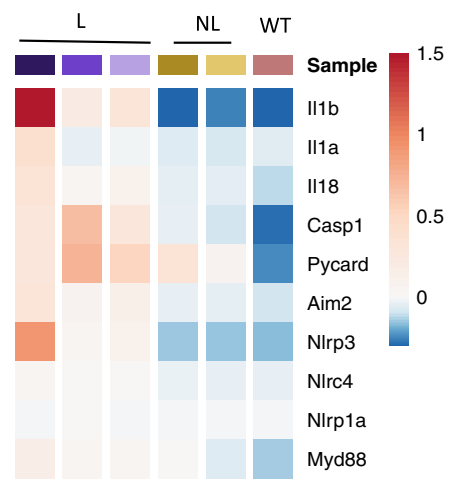

e.

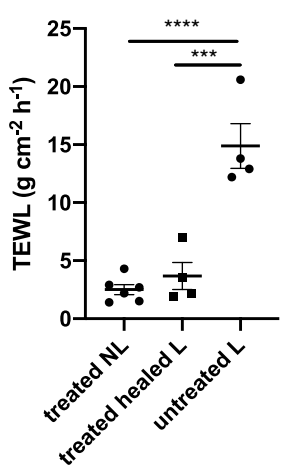

g.

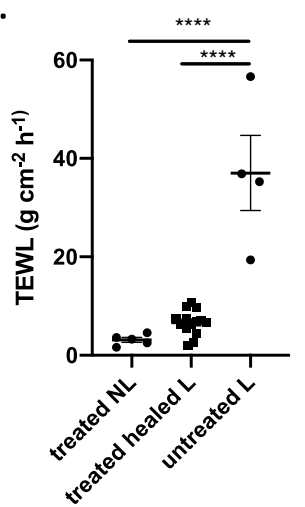

d.

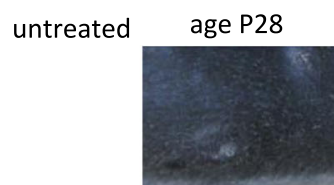

Anakinra

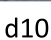

P41

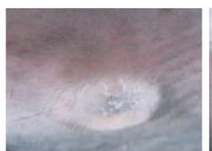

d23

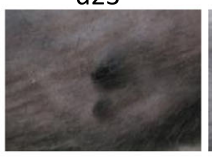

P47

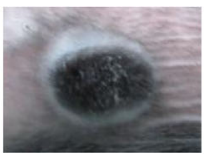

d29
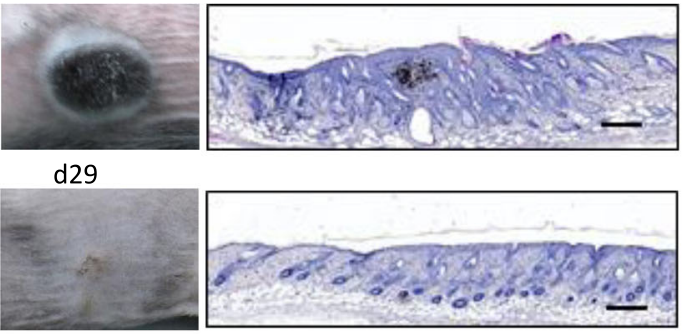

f.

$\alpha$ MCP-1

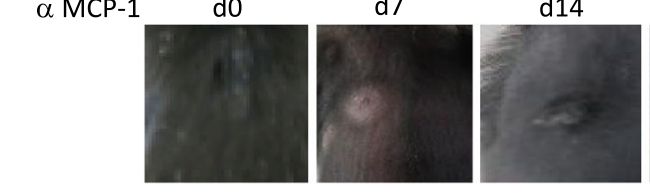

d7

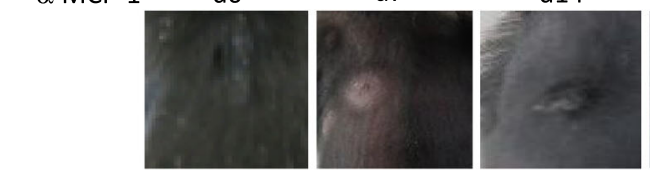

d14

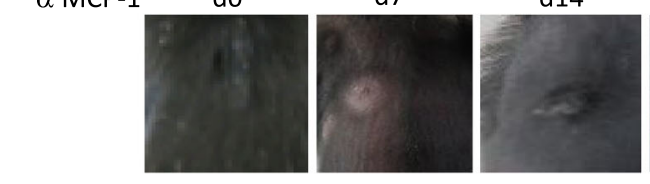

d21
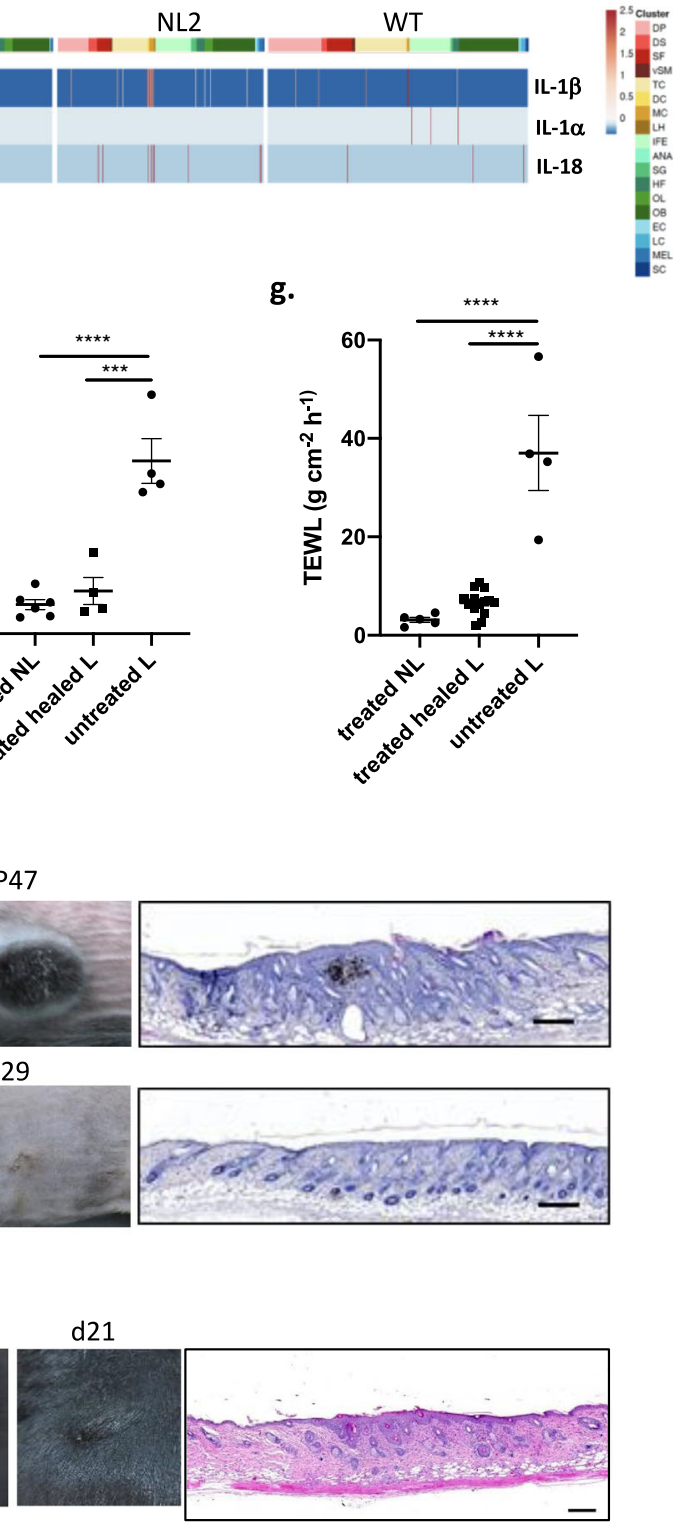

in different mouse tissues using the EBI expression atlas shows that Otulin expression strongly differs between different tissues with high expression in skin (Supplementary Fig. 7).

In conclusion, we have shown that linear deubiquitination of proteins by OTULIN serves as a crucial biological mechanism important for the maintenance of skin stem cell homeostasis and the prevention of keratinocyte death and subsequent skin inflammation. This further demonstrates that aberrant cell death can act as the driving force for tissue inflammation and neoplastic responses. In line with our observations, a study by the group of Manolis Pasparakis ${ }^{4}$, published in this issue of Nature Communications, describes similar findings, confirming the role of OTULIN in preventing skin inflammation by inhibiting the death of keratinocytes. 
Fig. 6 Interleukin-1 $\beta$ mediated signaling between innate immune cells and OTULIN-deficient keratinocytes regulates skin inflammation. a Schematic representation of NicheNet analysis identifying ligands secreted by innate immune cells that bind to receptors on keratinocytes, mediating changes in gene

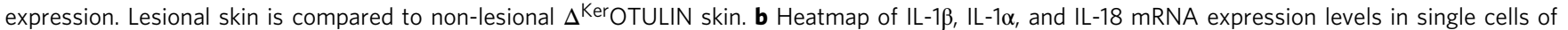

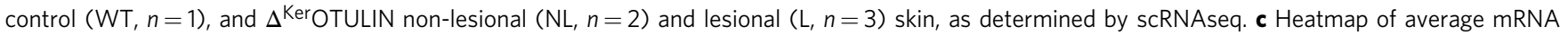

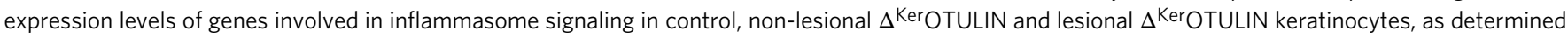
by scRNAseq analysis. d Representative pictures of back skin of mice treated with daily injections of $300 \mathrm{mg} / \mathrm{kg}$ Anakinra and untreated controls. Age of the mice is indicated and duration of treatment. H\&E-stained skin showing the morphology of the lesion at the time of sacrifice. Scale bar: $200 \mu \mathrm{m}$. e Transepidermal water loss (TEWL) measurements of non-lesional $(N L)$ and healed lesional $(L)$ skin of mice treated with Anakinra compared to untreated lesional skin (treated NL, $n=5$; healed $L$ and untreated $L n=4 ;{ }^{* \star *} p<0.001$; ${ }^{\star \star \star *} p<0.0001$, One-way ANOVA with multiple comparisons). Data represent means \pm SEM. $\mathbf{f}$ Representative pictures of back skin of mice treated with intraperitoneal injections of $40 \mathrm{mg} / \mathrm{kg} \alpha-M C P-1$ antibody twice weekly. Duration of treatment is indicated. H\&E-stained skin showing the morphology of the lesion at the time of sacrifice. Scale bar: $200 \mu \mathrm{m}$. $\mathbf{g}$ TEWL measurements of non-lesional (NL) and healed lesional ( $L$ ) skin of mice treated with $\alpha-M C P-1$ antibody compared to untreated lesional skin (treated NL, $n=5 ;$ healed $L$ and untreated $\mathrm{L} n=4 ;{ }^{\star \star \star} p<0.001 ;{ }^{\star \star \star \star} p<0.0001$, One-way ANOVA with multiple comparisons). Data represent means \pm SEM.

a.

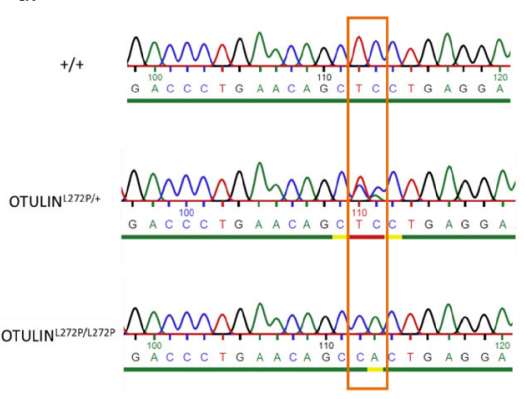

c.

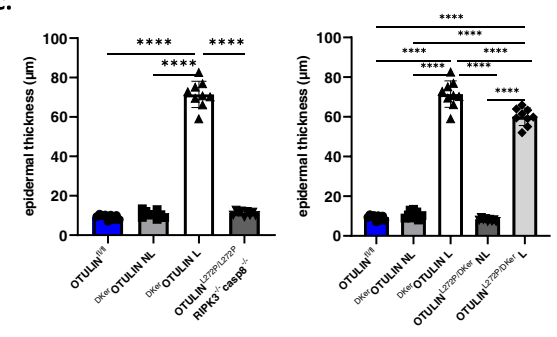

d.

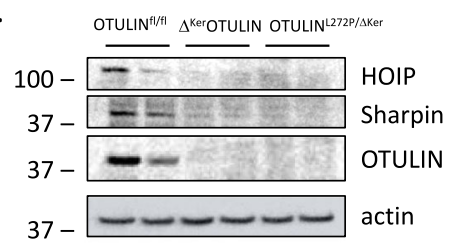

b.

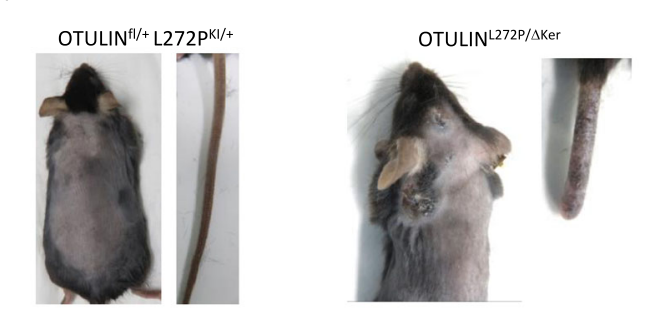

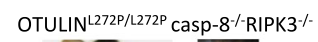
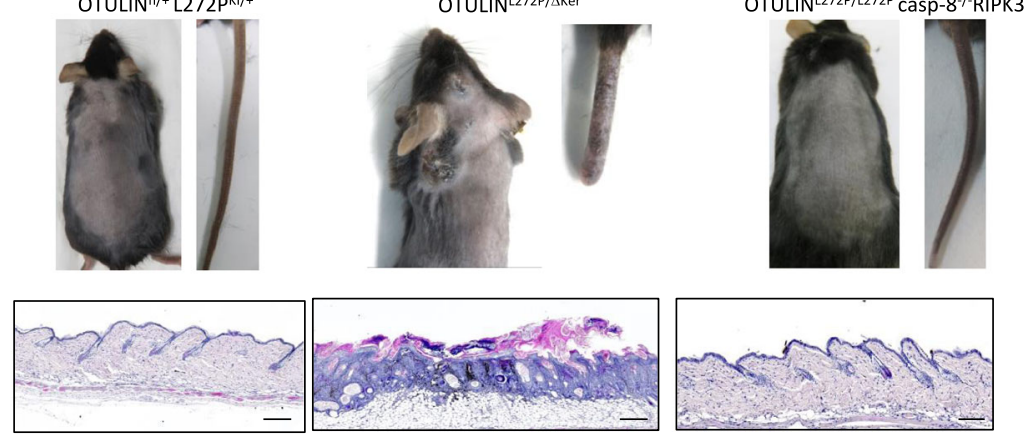

Fig. 7 Knockin of a human mutation in the murine Otulin gene phenocopies OTULIN-deficiency. a Targetting strategy for introduction of L272P mutation in the murine Otulin allele. b Representative images of the back skin and tail (upper panel), and H\&E-stained skin sections (lower panel) of OTULINL272P/+,

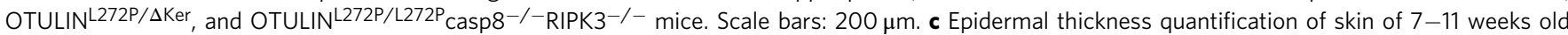
mice. NL non-lesional; L lesional ( $n=10$ per condition; ${ }^{\star \star \star \star} p<0.0001$, One-way ANOVA with multiple comparisons). Data represent means \pm SEM. d Immunoblotting on epidermal tail lysates of 7-week old OTULIN $\mathrm{N}^{\mathrm{fl} / \mathrm{fl}}(n=2), \Delta \operatorname{Ker}^{\mathrm{OTULIN}}(n=2)$ and OTULIN ${ }^{\text {L272P/DKer }}(n=2)$ mice using antibodies detecting HOIP, SHARPIN, and OTULIN. Anti-actin is shown as loading control. Molecular weight marker units are in kilodalton (kD).

\section{Methods}

Mice. The following mouse lines were used: Otulin ${ }^{F L 24}, \mathrm{K14}^{-C r e}{ }^{25}, \mathrm{Fadd}^{\mathrm{FL} 45}$,

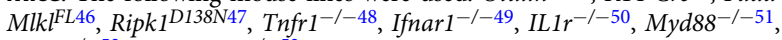
casp $8^{-/-52}$, and $R i p k 3^{-/-53}$. All alleles were maintained on a C57BL/6 genetic background. Mice were housed in individually-ventilated cages at the VIB Centre for Inflammation Research, in a specific pathogen-free animal facility at $20-24^{\circ} \mathrm{C}$ and $45-65 \%$ humidity with a $12 \mathrm{~h} \mathrm{light/dark} \mathrm{cycle} \mathrm{and} \mathrm{food} \mathrm{and} \mathrm{water} \mathrm{available} \mathrm{at}$ libitum. All experiments on mice were conducted according to institutional, national, and European animal regulations for animal testing and research. Animal protocols were approved by the VIB-Ghent University ethical review board.

Generation of OTULINL272P knockin mice. For the generation of OTULINL272P knockin mice, C57BL/6J zygotes were electroporated with $40 \mathrm{ng} / \mu \mathrm{l}$ Cas 9 protein (iDT), $8 \mathrm{ng} / \mu \mathrm{l} \mathrm{cr} /$ tracrRNA duplex (iDT) with guide sequence $5^{\prime}$ ATGACCCT
GAACAGCTCCTG $3^{\prime}$ and $150 \mathrm{ng} / \mu \mathrm{l}$ single-stranded DNA repair template with sequence 5'- AAGTGCCGTTCTTCTCTGTGCTCTTGTTTGCCCGAGACACA TCCAATGACCCTGAACAGCCACTGAGGAACCACCTAAACCAGGTGGGAC ACACGGGGGGCCTTGAGCAGGTGAGTTGTGGC-3' (iDT) containing the L272P mutation (CTC > CCA). The embryos were transferred to foster mothers the same day through oviduct transfer. Effective mutagenesis was confirmed by sequencing of the Otulin gene on genomic DNA.

Therapeutic treatment studies. $\Delta^{\mathrm{Ker} O T U L I N}$ mice were either or not treated with $300 \mathrm{mg} / \mathrm{kg}$ Anakinra (Sobi, Kineret $100 \mathrm{mg} / 0.67 \mathrm{ml}$ ) by intraperitoneal (IP) injection daily from the age of 18 days on and for a period of 30 days, or with $40 \mathrm{mg} / \mathrm{kg}$ anti-MCP-1 antibody (InVivoMab, BE0185) IP twice a week from the age of 18 days on and for a period of 30 days. 
Table 1. depicting the number of offspring from the indicated intercrosses (observed and expected ratios).

\begin{tabular}{lll}
$\begin{array}{l}\text { OTULINL272P/ }+ \\
\text { OTULIN }\end{array}$-272P/+ & Expected & $\begin{array}{l}\text { Observed } \\
\text { (at birth) }\end{array}$ \\
\hline OTULIN $+/+$ & $25 \%$ & $34 \%(31)$ \\
OTULINL272P/+ & $(26-27)$ & \\
OTULINL272P/L272P & $50 \%(53)$ & $66 \%(75)$ \\
& $25 \%$ & $0 \%(0)$ \\
Total (observed) & $(26-27)$ & \\
\hline
\end{tabular}

\begin{tabular}{|c|c|c|}
\hline $\begin{array}{l}\text { OTULIN }{ }^{L 272 P /+} \text { Casp-8 }^{-/-} \\
\text {RIPK3 }^{-/-} \text {X } \\
\text { OTULIN }^{\text {L272P/+ }} \text { Casp-8 } \\
\text { RIPK3 }^{-/-}\end{array}$ & Expected & $\begin{array}{l}\text { Observed (at } 8 \text { weeks } \\
\text { of age) }\end{array}$ \\
\hline OTULIN $+/+$ & $\begin{array}{l}25 \% \\
(13-14)\end{array}$ & $22 \%(12)$ \\
\hline OTULINL272P/+ & $50 \%(27)$ & $54 \%(29)$ \\
\hline OTULINL272P/L272P & $\begin{array}{l}25 \% \\
(13-14)\end{array}$ & $24 \%(13)$ \\
\hline Total (observed) & $100 \%(54)$ & $100 \%(54)$ \\
\hline
\end{tabular}

Wound healing assay. Full-thickness wounds on the back skin of shaved mice were made by using an $8 \mathrm{~mm}$ punch biopsy needle (Stiefel Instruments) under analgesia and general anesthesia in 7 weeks-old transgenic and control littermates. Wound sizes were measured every other day by two independent researchers, who were blinded to group allocations.

Primary keratinocytes. Primary mouse keratinocytes were isolated from $\Delta^{\text {Ker- }}$ OTULIN and OTULIN ${ }^{\mathrm{fl} / \mathrm{fl}}$ skin as previously described ${ }^{54}$. Briefly, shaved back skin was isolated, sterilized, and floated on $0.25 \%$ trypsin overnight. The epidermis was separated from dermis and cultured on confluent feeder cultures.

Flow cytometry. Immunophenotyping of mouse skin was performed on single-cell suspensions obtained following trypsin digestion for $1 \mathrm{~h}$ at $37^{\circ} \mathrm{C}$ and subsequent digestion with collagenase type-1 $(1.25 \mathrm{mg} / \mathrm{ml}$; GIBCO), type- $2(0.5 \mathrm{mg} / \mathrm{ml}$, Sigma $)$ and type $-4(0.5 \mathrm{mg} / \mathrm{ml}$, Sigma $)$ for $30-45 \mathrm{~min}$. Cells were stained with the following fluorochrome-linked antibodies, as listed in Supplementary Table 1. Prior to measuring, counting beads (Life Technologies) were added to the cells. Measurements were performed on a BD Fortessa 5-laser cytometer and analyzed using FlowJo 10.6.1. software (Tree Star).

Immunofluorescence. Dewaxed paraffin or frozen skin sections or horizontal tail wholemounts were labeled with respective antibodies listed in Supplementary Table 1. As secondary antibodies donkey-anti-mouse 488 AlexaFluor (1:2000) and goat-anti-rabbit DyLight 586 (1:2000) were used in combination with Dapi.

Immunohistochemistry. Dewaxed paraffin skin sections were subjected to heatmediated antigen retrieval (citrate buffer; $\mathrm{pH}=6$ ) and labeled with $\mathrm{Ki}-67 \mathrm{Ab}(1$ 1000; Cell Signaling Technology, D3B5, 12202) or cleaved caspase-3 Ab (1: 1000; Cell Signaling Technology, 9661). Slides were incubated with secondary antibody followed by avidin-biotin complexes and peroxidase activity was detected with diaminobutyric acid (DAB) substrate (Vector Laboratories).

EdU tracing. Mice were intraperitoneally injected with EdU $(1.25 \mathrm{mg} / \mathrm{kg}$ in PBS Click-IT EdU Alexa 488 Imaging Kit) $3 \mathrm{~h}$ prior to sacrifice. Staining was performed according to the manufacturers' instructions.

Transepidermal water loss (TEWL) measurements. Mice were shaved one day prior to TEWL assessment. TEWL was measured on the back skin of anesthetized mice using a TEWA meter (courage and Khazaka, TM 210).

Western blot analysis. Primary keratinocytes and skin tissue were homogenized using E1A lysis buffer (50 mM HEPES pH7.6; $250 \mathrm{mM} \mathrm{NaCl} ; 5 \mathrm{mM}$ EDTA; 0.5\% NP40) and NP-40 (50 mM Tris-HCl, pH 7.6; 1 mM EDTA; $150 \mathrm{mM} \mathrm{NaCl} ; 1 \%$ NP$40 ; 0.5 \%$ sodiumdeoxycholate; $0.1 \%$ SDS) buffer respectively containing protease inhibitors (Roche) and phosphatase inhibitors (Sigma), denaturated in $1 \times$

Laemmli buffer (50 mM Tris-HCl pH8.2; $2 \%$ SDS; $10 \%$ glycerol; $0.005 \%$ BFB; $5 \%$ $\beta$-mercapto-ethanol) and boiled for $10 \mathrm{~min}$ at $95^{\circ} \mathrm{C} .20 \mu \mathrm{g}$ of liver lysates and $20 \mu \mathrm{g}$ of cell lysates were separated by SDS-polyacrylamide gel electrophoresis (PAGE), transferred to nitrocellulose and analyzed by immunoblotting. Membranes were probed with antibodies against OTULIN (1:1000, Cell Signaling Technology, 14127), JNK (1:1000, BD Bioscience, 554285), phospho-JNK (1:1000, Millipore,

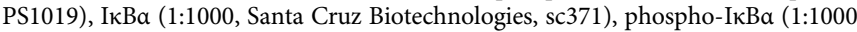
Cell Signaling Technology, 9246), p38 MAPK (1:1000, Cell Signaling Technology CST9212), phospho-p38 MAPK (1:1000, Cell Signaling Technology, CST9215), HOIL-1 (1:2000, kind gift of Dr. Henning Walczak, UCL London), HOIP (1:1000, kind gift of Dr. Rune Damgaard, MRC Cambridge), SHARPIN (1:1000, Proteintech, 14626-1-AP), Ripk1 (1:2000, Cell Signaling Technology, 3493), linear ubiquitin (1:2500, Millipore, clone LUB9, MABS451), caspase-3 (1:1000; Cell Signaling, 9662) and actin-HRP (1:10000, MP Biomedicals). As secondary antibodies, HRP coupled anti-rabbit-HRP, anti-mouse-HRP, and anti-goat-HRP were used (1:2500, Amersham) and detection was done by chemiluminescence (Western Lightning Plus ECL, Perkin Elmer) using the Amersham Imager 600 (GE Healthcare).

Immunoprecipitation. Recombinant GST-UBAN was produced in BL21(DE3) cells. In brief, BL21(DE3) cells were transformed with the plasmid encoding GSTUBAN and protein expression was induced with $0.5 \mathrm{M}$ IPTG. After $4 \mathrm{~h}$, cells were collected and lysed in lysis buffer (20 mM Tris-HCl pH 7.5, $10 \mathrm{mM}$ EDTA, $5 \mathrm{mM}$ EGTA, $150 \mathrm{mM} \mathrm{NaCl}, 1 \mathrm{mM}$ DTT supplemented with phosphatase and protease inhibitor cocktail tablets (Roche Diagnostics)), sonicated, and cleared by centrifugation. After centrifugation, Triton-X100 (0.5\% final concentration) was added to the supernatant, which was then transferred onto prewashed glutathione beads and left rotating for $2 \mathrm{~h}$ at $4{ }^{\circ} \mathrm{C}$. After incubation, the beads were centrifuged, washed twice with washing buffer $(20 \mathrm{mM}$ Tris- $\mathrm{HCl}$ pH 7.5, $10 \mathrm{mM}$ EDTA, $150 \mathrm{mM} \mathrm{NaCl}, 0.5 \%$ Triton-X100) and resuspended in resuspension buffer $(20 \mathrm{mM}$ Tris- $\mathrm{HCl} \mathrm{pH}$ 7.5, $0.1 \% \beta$-mercaptoethanol, $0.05 \%$ sodiumazide), ready to be used. Cell lysates from total skin tissue were prepared as described before, protein concentration was determined and $800 \mu \mathrm{g}$ protein lysate was incubated overnight with GST-UBAN-containing glutathione beads. The next day, the beads were washed three times in RIPA lysis buffer $(150 \mathrm{mM} \mathrm{NaCl}, 1 \% \mathrm{NP}-40,0.5 \%$ Sodium Deoxycholate, $0.1 \%$ SDS, $10 \mathrm{mM}$ Tris- $\mathrm{HCl} \mathrm{pH} 8$ supplemented with phosphatase and protease inhibitor cocktail tablets (Roche Diagnostics)). Beads were then resuspended in $60 \mu \mathrm{L} 1 \times$ laemmli buffer for direct analysis.

Real-time RT PCR. Total RNA was isolated using TRIzol reagent (Invitrogen) and Aurum Total RNA Isolation Mini Kit (Biorad), according to the manufacturer's instructions. Synthesis of cDNA was performed using Sensifast cDNA Synthesis Kit (Bioline) according to the manufacturer's instructions. cDNA was amplified on quantitative PCR in a total volume of $5 \mu \mathrm{l}$ with SensiFAST SYBR ${ }^{\circledR}$ No-ROX Kit (Bioline) and specific primers on a LightCycler 480 (Roche). The reactions were performed in duplicates. The mouse-specific primers used are summarized in Supplementary Table 2 .

Cytokine detection. Cytokine concentrations in serum were determined by a magnetic bead-based multiplex assay using Luminex technology (BioRad), according to the manufacturer's instructions. Cytokine concentrations observed in $\mathrm{OTULIN}^{\mathrm{fl} / \mathrm{fl}}$ and ${ }^{\Delta \mathrm{Ker}} \mathrm{OTULIN}$ mice were re-used in figure panels $1 \mathrm{~g}, 3 \mathrm{~b}$, and $5 \mathrm{e}$.

scRNA sequencing and analysis. Single-cell suspensions were obtained from total mouse skin as previously described ${ }^{55}$. Live cells were sorted on FACS Aria by gating for L/D eFluor780 negative cells into PBS with $0.04 \%$ BSA, spun down, and resuspended in PBS with $0.04 \%$ BSA at a final concentration of 1000 cells/ $\mu$ l. Cellular suspensions (target recovery of 10,000 cells) were loaded on a GemCode Single-Cell Instrument (10x Genomics, Pleasanton) to generate single-cell Gel Bead-in-Emulsion (GEMs). Single-cell RNA-Seq libraries were prepared using GemCode Single-Cell V2 3'Gel Bead and Library kit (10x Genomics) according to the manufacturer's instructions. Sequencing libraries were loaded at $2.1 \mathrm{pM}$ loading concentration on a HiSeq 4000 with custom sequencing metrics (single-indexed sequencing run, 28/8/0/98 cycles for R1/i7/i5/R2) (Illumina, San Diego, CA). Sequencing was performed at the VIB Nucleomics Core (VIB, Leuven, Belgium). Demultiplexing of the raw data and mapping to the mouse genome mm 10 (1.2.0) was done by the 10X CellRanger software (version 2.1.1; cellranger). Preprocessing of the data was done by the scran and scater R package (version 4.1) according to the workflow proposed by the Marioni and Theis lab $^{34}$. Outlier cells were identified based on 3 metrics (library size, number of expressed genes, and mitochondrial proportion) and cells were tagged as outliers when they were a certain median absolute deviation (MAD) away from the median value of each metric across all cells. For the library size a lower bound of 4 and upper bound of 3 MADs were used, with exception of samples NL1, L3 (upper bound of 2,5 MADs) and WT (upper bound of 4 MADs). For the number of expressed genes the value was set to 4 MADs for both the upper and lower bound, with exception of samples L1, L2 (upper bound of 3 MADs) and L3 (upper bound of 2 MADs). For the mitochondrial proportion 10 MADs were used in samples L1, L2, and NL1, 15 MADs in L3 and 8 MADs in WT. Low-quality cells (low UMI counts, high percentage of mitochondrial genes) were removed from the analysis. A principal component analysis (PCA) plot was generated as an extra filtering using the runPCA function from the scater R package with the default parameters. Genes expressed in less than 3 cells and cells expressing less than 200 genes were removed. The samples were 
aggregated using the merge function, counts were normalized and $\log 2$ transformed using the NormalizeData function, both from the Seurat R package (v3.1.0) using default parameters. Detecting highly variable genes, finding clusters, and creating UMAP plots was done using the Seurat pipeline. Clustering was performed using the first 34 principal components and a resolution of 0.8 .

Statistics. Results are expressed as means \pm SEM. Statistical significance between OTULIN $^{\mathrm{l} / \mathrm{fl}}$ and $\Delta^{\mathrm{Ker}}$ OTULIN was assessed using a Mann-Whitney testing, Oneway ANOVA, or Two-way ANOVA with multiple comparisons. Statistical significance between $\Delta^{\mathrm{Ker}}$ OTULIN and the different genetic crosses was assessed using a one-way ANOVA test, followed by Tukey's multiple comparison test. The analysis was performed with Prism 9 software. To compare the percentages of sytox green positive cells, we analyzed repeated measurements using the method of residual maximum likelihood (REML), as implemented in Genstat version 21. When representative images are shown, a minimum of 12 mice of the relevant genotype were analyzed. Comparison of HFSC marker positive cells in the permanent epidermis was done by Chi-square testing, comparing L or NL gene fre quencies to expected frequencies from WT.

Reporting summary. Further information on research design is available in the Nature Research Reporting Summary linked to this article.

\section{Data availability}

Source data are provided with this paper. The accession number for the raw scRNAsequencing data reported in this study is Gene Expression Omnibus $(\mathrm{GeO})$ : GSE162394. Source data are provided with this paper.

Received: 7 November 2020; Accepted: 9 September 2021; Published online: 08 October 2021

\section{References}

1. Arwert, E. N., Hoste, E. \& Watt, F. M. Epithelial stem cells, wound healing and cancer. Nat. Rev. Cancer 12, 170-180 (2012).

2. Ge, Y. \& Fuchs, E. Stretching the limits: from homeostasis to stem cell plasticity in wound healing and cancer. Nat. Rev. Genet. 19, 311-325 (2018).

3. Joost, S. et al. Single-cell transcriptomics of traced epidermal and hair follicle stem cells reveals rapid adaptations during wound healing. Cell Rep. 25, 585-597 (2018).

4. Peltzer, N. et al. LUBAC is essential for embryogenesis by preventing cell death and enabling haematopoiesis. Nature 557, 112-117 (2018).

5. Gerlach, B. et al. Linear ubiquitination prevents inflammation and regulates immune signalling. Nature 471, 591-596 (2011).

6. Ikeda, F. et al. SHARPIN forms a linear ubiquitin ligase complex regulating NF-kappaB activity and apoptosis. Nature 471, 637-641 (2011)

7. Tokunaga, F. et al. SHARPIN is a component of the NF-kappaB-activating linear ubiquitin chain assembly complex. Nature 471, 633-636 (2011).

8. Taraborrelli, L. et al. LUBAC prevents lethal dermatitis by inhibiting cell death induced by TNF, TRAIL, and CD95L. Nat. Commun. 9, 3910 (2018).

9. HogenEsch, H. et al. A spontaneous mutation characterized by chronic proliferative dermatitis in C57BL mice. Am. J. Pathol. 143, 972-982 (1993).

10. Seymour, R. E. et al. Spontaneous mutations in the mouse Sharpin gene result in multiorgan inflammation, immune system dysregulation, and dermatitis. Genes Immun. 8, 416-421 (2007).

11. Peltzer, N. et al. HOIP deficiency causes embryonic lethality by aberrant TNFR1-mediated endothelial cell death. Cell Rep. 9, 153-165 (2014).

12. Keusekotten, $\mathrm{K}$. et al. OTULIN antagonizes LUBAC signaling by specifically hydrolyzing Met1-linked polyubiquitin. Cell 153, 1312-1326 (2013).

13. Rivkin, E. et al. The linear ubiquitin-specific deubiquitinase gumby regulates angiogenesis. Nature 498, 318-324 (2013).

14. Verboom, L., Hoste, E. \& van Loo, G. OTULIN in NF-kappaB signaling, cell death, and disease. Trends Immunol. 42, P590-603 (2021).

15. Damgaard, R. B. et al. The deubiquitinase OTULIN is an essential negative regulator of inflammation and autoimmunity. Cell 166, 1215-1230 (2016).

16. Zhou, Q. et al. Biallelic hypomorphic mutations in a linear deubiquitinase define otulipenia, an early-onset autoinflammatory disease. Proc. Natl Acad. Sci. USA 113, 10127-10132 (2016).

17. Heger, K. et al. OTULIN limits cell death and inflammation by deubiquitinating LUBAC. Nature 559, 120-124 (2018).

18. Elliott, P. R. et al. Molecular basis and regulation of OTULIN-LUBAC interaction. Mol. Cell 54, 335-348 (2014).

19. Draber, P. et al. LUBAC-recruited CYLD and A20 regulate gene activation and cell death by exerting opposing effects on linear ubiquitin in signaling complexes. Cell Rep. 13, 2258-2272 (2015).
20. Schaeffer, V. et al. Binding of OTULIN to the PUB domain of HOIP controls NF-kappaB signaling. Mol. Cell 54, 349-361 (2014).

21. Peltzer, N. \& Walczak, H. Cell death and inflammation-a vital but dangerous liaison. Trends Immunol. 40, 387-402 (2019).

22. Ting, A. T. \& Bertrand, M. J. M. More to Life than NF-kappaB in TNFR1 Signaling. Trends Immunol. 37, 535-545 (2016).

23. Kumari, S. \& Pasparakis, M. Epithelial cell death and inflammation in skin. Curr. Top. Microbiol. Immunol. 403, 77-93 (2017).

24. Verboom, L. et al. OTULIN prevents liver inflammation and hepatocellular carcinoma by inhibiting FADD- and RIPK1 kinase-mediated hepatocyte apoptosis. Cell Rep. 30, 2237-2247 (2020).

25. Hafner, M. et al. Keratin 14 Cre transgenic mice authenticate keratin 14 as an oocyte-expressed protein. Genesis 38, 176-181 (2004).

26. Santoro, A. et al. A troubling diagnosis of verrucous squamous cell carcinoma ("the Bad Kind" of Keratosis) and the need of clinical and pathological correlations: a review of the literature with a case report. J. Skin Cancer 2011 370605 (2011)

27. Strid, J., McLean, W. H. I. \& Irvine, A. D. Too much, too little or just enough: a goldilocks effect for IL-13 and skin barrier regulation? J. Invest. Dermatol. 136, 561-564 (2016).

28. Damgaard, R. B. et al. OTULIN protects the liver against cell death, inflammation, fibrosis, and cancer. Cell Death Differ. 27, 1457-1474 (2020).

29. Damgaard, R. B. et al. OTULIN deficiency in ORAS causes cell type-specific LUBAC degradation, dysregulated TNF signalling and cell death. EMBO Mol. Med. 11, e9324 (2019).

30. Rickard, J. A. et al. TNFR1-dependent cell death drives inflammation in Sharpin-deficient mice. Elife 3, e03464 (2014).

31. Kumari, S. et al. Sharpin prevents skin inflammation by inhibiting TNFR1induced keratinocyte apoptosis. Elife 3, e03422 (2014).

32. Pasparakis, M. \& Vandenabeele, P. Necroptosis and its role in inflammation. Nature 517, 311-320 (2015)

33. Liang, Y. \& Sundberg, J. P. SHARPIN regulates mitochondria-dependent apoptosis in keratinocytes. J. Dermatol. Sci. 63, 148-153 (2011).

34. Lun, A. T., McCarthy, D. J. \& Marioni, J. C. A step-by-step workflow for lowlevel analysis of single-cell RNA-seq data with Bioconductor. F1000Res. 5, 2122 (2016).

35. Joost, S. et al. The molecular anatomy of mouse skin during hair growth and rest. Cell Stem Cell 26, 441-457 (2020).

36. Browaeys, R., Saelens, W. \& Saeys, Y. NicheNet: modeling intercellular communication by linking ligands to target genes. Nat. Methods 17, 159-162 (2020).

37. Dinarello, C. A. \& Thompson, R. C. Blocking IL-1: interleukin 1 receptor antagonist in vivo and in vitro. Immunol. Today 12, 404-410 (1991).

38. Nabavi, M. et al. Auto-inflammation in a patient with a novel homozygous OTULIN mutation. J. Clin. Immunol. 39, 138-141 (2019).

39. Kaiser, W. J. et al. RIP3 mediates the embryonic lethality of caspase-8deficient mice. Nature 471, 368-372 (2011)

40. Oberst, A. et al. Catalytic activity of the caspase-8-FLIP(L) complex inhibits RIPK3-dependent necrosis. Nature 471, 363-367 (2011).

41. Gough, D. J. et al. Constitutive type I interferon modulates homeostatic balance through tonic signaling. Immunity 36, 166-174 (2012).

42. Gui, J. et al. Therapeutic elimination of the type 1 interferon receptor for treating psoriatic skin inflammation. J. Invest. Dermatol. 136, 1990-2002 (2016).

43. Grine, L. et al. Dual inhibition of TNFR1 and IFNAR1 in imiquimod-induced psoriasiform skin inflammation in mice. J. Immunol. 194, 5094-5102 (2015).

44. Schünke, H., Goebel, U., Dikic, I. \& Pasparakis, M. OTULIN inhibits RIPK1mediated keratinocyte necroptosis to prevent skin inflammation in mice. Nat Commun. https://doi.org/10.1038/s41467-021-25945-1 (2021).

45. Mc Guire, C. et al. Oligodendrocyte-specific FADD deletion protects mice from autoimmune-mediated demyelination. J. Immunol. 185, 7646-7653 (2010).

46. Murphy, J. M. et al. The pseudokinase MLKL mediates necroptosis via a molecular switch mechanism. Immunity 39, 443-453 (2013).

47. Polykratis, A. et al. Cutting edge: RIPK1 kinase inactive mice are viable and protected from TNF-induced necroptosis in vivo. J. Immunol. 193, 1539-1543 (2014).

48. Pfeffer, K. et al. Mice deficient for the $55 \mathrm{kd}$ tumor necrosis factor receptor are resistant to endotoxic shock, yet succumb to L. monocytogenes infection. Cell 73, 457-467 (1993).

49. Muller, U. et al. Functional role of type I and type II interferons in antiviral defense. Science 264, 1918-1921 (1994).

50. Labow, M. et al. Absence of IL-1 signaling and reduced inflammatory response in IL-1 type I receptor-deficient mice. J. Immunol. 159, 2452-2461 (1997).

51. Adachi, O. et al. Targeted disruption of the MyD88 gene results in loss of IL1- and IL-18-mediated function. Immunity 9, 143-150 (1998).

52. Salmena, L. et al. Essential role for caspase 8 in T-cell homeostasis and T-cellmediated immunity. Genes Dev. 17, 883-895 (2003). 
53. Newton, K., Sun, X. \& Dixit, V. M. Kinase RIP3 is dispensable for normal NFkappa Bs, signaling by the B-cell and T-cell receptors, tumor necrosis factor receptor 1, and Toll-like receptors 2 and 4. Mol. Cell Biol. 24, 1464-1469 (2004).

54. Jensen, K. B., Driskell, R. R. \& Watt, F. M. Assaying proliferation and differentiation capacity of stem cells using disaggregated adult mouse epidermis. Nat. Protoc. 5, 898-911 (2010).

55. Hoste, E. et al. Epithelial HMGB1 delays skin wound healing and drives tumor initiation by priming neutrophils for NET formation. Cell Rep. 29, 2689-2701 (2019).

\section{Acknowledgements}

We thank the EUCOMM Consortium for Otulin-targeted ES cells, Manolis Pasparakis for providing RIPK1 ${ }^{\mathrm{D} 138 \mathrm{~N}}$ mice, Vishva Dixit and Genentech for providing RIPK3 ${ }^{-1-}$ mice, and Alexander Warren and James Murphy (The Walter and Eliza Hall Institute of Medical Research, Melbourne, Australia) for the use of floxed Mlkl mice. We thank Sofie De Schepper for pathological assessment of $\Delta^{\mathrm{Ker} O T U L I N}$ mice, and Laetitia Bellen, Dimitri Huygebaert and Dieter Vanhede for animal care. We acknowledge helpful input from Jonathan Maelfait and the VIB BioImaging Core, the VIB Nucleomics Core, and the VIB Flowcore for technical assistance, and all members of the van Loo lab for suggestions and discussions. E.H. is supported by an FWO postdoctoral fellowship and a CRIG Young-Investigator grant. A.M. is supported by an FWO postdoctoral fellowship, L.v.H and L.V. are supported by an FWO FR fellowship. K.R. holds an Odysseus Grant and ERC Advanced grant. Research in the van Loo lab is financed by research grants from the FWO, Stichting Tegen Kanker, the Charcot Foundation, Kom op Tegen Kanker and the "Concerted Research Actions" (GOA) of the Ghent University.

\section{Author contributions}

E.H.: conception and design of experiments, analysis and acquisition of data, drafting and revising the paper. K.L.: experimental design, analysis and acquisition of data, drafting and revising the paper. K.A., N.v.D., J.R., M.S., A.M., L.v.H., L.V., S.M., K.V., H.K.V., K.R., Y.S., and M.K.: analysis and acquisition of data. T.H.: generation of OTULIN $^{\text {L272P }}$ knockin mice. G.v.L.: conception and design of experiments, data analysis, drafting, and revising the paper.

\section{Competing interests}

The authors declare no competing interests.

\section{Additional information}

Supplementary information The online version contains supplementary material available at https://doi.org/10.1038/s41467-021-25944-2.

Correspondence and requests for materials should be addressed to Esther Hoste or Geert van Loo.

Peer review information Nature Communications thanks Mads Gyrd-Hansen and ShaoCong Sun for their contribution to the peer review of this work. Peer reviewer reports are available.

Reprints and permission information is available at http://www.nature.com/reprints

Publisher's note Springer Nature remains neutral with regard to jurisdictional claims in published maps and institutional affiliations.

(c) (i) Open Access This article is licensed under a Creative Commons Attribution 4.0 International License, which permits use, sharing, adaptation, distribution and reproduction in any medium or format, as long as you give appropriate credit to the original author(s) and the source, provide a link to the Creative Commons license, and indicate if changes were made. The images or other third party material in this article are included in the article's Creative Commons license, unless indicated otherwise in a credit line to the material. If material is not included in the article's Creative Commons license and your intended use is not permitted by statutory regulation or exceeds the permitted use, you will need to obtain permission directly from the copyright holder. To view a copy of this license, visit http://creativecommons.org/ licenses/by/4.0/.

(c) The Author(s) 2021 